\title{
Scattering of magnetic edge states
}

\section{Journal Article}

\section{Author(s):}

Buchendorfer, Christoph; Graf, Gian M.

Publication date:

2006-03

Permanent link:

https://doi.org/10.3929/ethz-b-000000354

Rights / license:

In Copyright - Non-Commercial Use Permitted

Originally published in:

Annales Henri Poincaré 7(2), https://doi.org/10.1007/s00023-005-0250-8 


\title{
Scattering of Magnetic Edge States
}

\author{
Christoph Buchendorfer and Gian Michele Graf
}

\begin{abstract}
We consider a charged particle following the boundary of a two dimensional domain because a homogeneous magnetic field is applied. We develop the basic scattering theory for the corresponding quantum mechanical edge states. The scattering operator attains a limit for large magnetic fields which preserves Landau bands. We interpret the corresponding scattering phases in terms of classical trajectories.
\end{abstract}

\section{Introduction}

A charged particle moving in a domain $\Omega \subset \mathbb{R}^{2}$ under the influence of a homogeneous magnetic field $B$ may follow a skipping orbit along the boundary $\partial \Omega$. The quantum mechanical counterpart to these orbits are extended chiral states supported near $\partial \Omega$. Under certain geometric conditions these states give rise to some purely absolutely continuous spectrum [7] at energies $E$ away from the Landau levels associated with bulk states, i.e., at $E \in B \cdot \Delta$ with

$$
\bar{\Delta} \cap(2 \mathbb{N}+1)=\emptyset
$$

This work is about the scattering of such chiral edge states at a bent of an otherwise straight boundary $\partial \Omega$. Being chiral they never backscatter, but may undergo transitions between different bands. The main results are that such transitions are suppressed at large $B$ fields and that, within each band, the edge states acquire an additional phase as compared to particles following a straight boundary of the same length. That phase is proportional to the bending angle but independent of the (large) magnetic field. We remark that the scattering of edge states is at the basis of some theories of the quantum Hall effect [4].

The precise formulation of the setup and of the results requires some preliminaries. We consider a simply connected domain $\Omega \subset \mathbb{R}^{2}$ with oriented boundary $\partial \Omega$ consisting of a single, unbounded smooth curve $\gamma \in C^{4}(\mathbb{R})$ parameterized by arc length $s \in \mathbb{R}$. We assume that $\gamma$ is eventually straight in the sense that the curvature $\kappa(s)=\dot{\gamma}(s) \wedge \ddot{\gamma}(s) \in \mathbb{R},(\cdot=\mathrm{d} / \mathrm{d} s)$, is compactly supported. The bending angle

$$
\theta:=\int_{-\infty}^{\infty} \kappa(s) \mathrm{d} s
$$


takes values in $[-\pi, \pi]$ and we assume

$$
\theta \neq \pi,
$$

which ensures that $\Omega$ contains a wedge of positive opening angle $\pi-\theta$.

Since the cyclotron radius, and hence the lateral extent of an edge state, scales as $B^{-1 / 2}$, it will be notationally convenient to represent the homogeneous field as $B=\beta^{2}$. The Hamiltonian is

$$
H=B^{-1}(-i \nabla-B A(x))^{2}=\left(-i \beta^{-1} \nabla-\beta A(x)\right)^{2}
$$

on $\mathcal{H}:=L^{2}(\Omega)$ with Dirichlet boundary conditions on $\partial \Omega$. Here $A: \Omega \rightarrow \mathbb{R}^{2}$ is a gauge field producing a unit magnetic field, $\partial_{1} A_{2}-\partial_{2} A_{1}=1$. This is the usual magnetic Hamiltonian except for a rescaling of energy, which is now measured in units of Landau levels spacings. This, or the equivalent rescaling of time, does not affect the scattering operator, but will simplify its analysis.

As the dynamics of the edge states is effectively one-dimensional, it is natural to eliminate the gauge field from its description. For the 2-dimensional system this means that we restrict to gauges with $A_{\|}=0$ on $\partial \Omega$, i.e.,

$$
A(\gamma(s)) \cdot \dot{\gamma}(s)=0 .
$$

A particle moving in a half-plane $\Omega_{0}=\mathbb{R} \times \mathbb{R}_{+} \ni(s, u)$ will serve as a model for the asymptotic dynamics, both in the past (or at $s \rightarrow-\infty$ ) and in the future (or at $s \rightarrow+\infty$ ). We denote the corresponding Hamiltonian on $\mathcal{H}_{0}:=L^{2}\left(\Omega_{0}\right)$ by

$$
H_{0}:=\left(-i \beta^{-1} \partial_{s}+\beta u\right)^{2}+\left(-i \beta^{-1} \partial_{u}\right)^{2},
$$

where we have used the Landau gauge $A=(-u, 0)$.

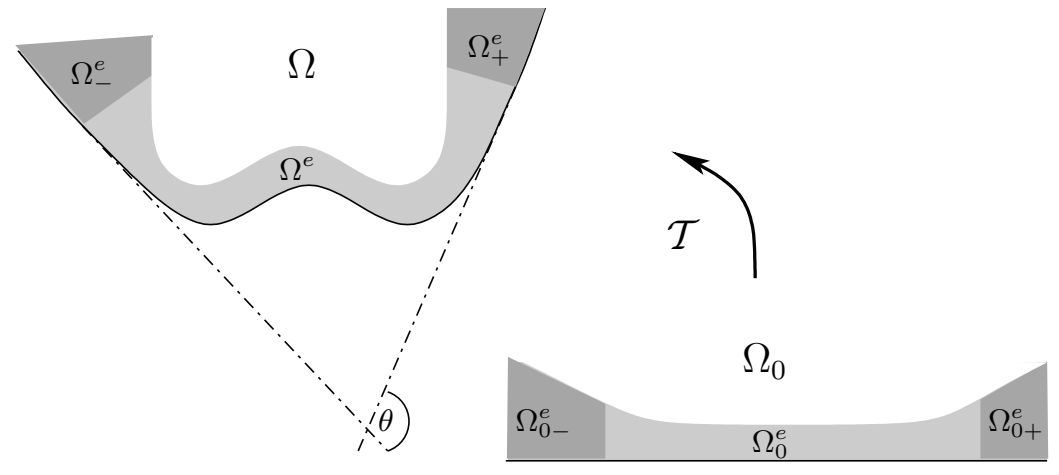

Figure 1. Left: The domains $\Omega, \Omega^{e}, \Omega_{ \pm}^{e}$.

Figure 2. Right: The domains $\Omega_{0}, \Omega_{0}^{e}, \Omega_{0 \pm}^{e}$. 
To serve as scattering asymptotes, states in $L^{2}\left(\Omega_{0}\right)$ have to be identified with states in $L^{2}(\Omega)$. To this end we introduce the tubular map:

$$
\begin{gathered}
\mathcal{T}: \Omega_{0} \rightarrow \mathbb{R}^{2} \\
x(s, u) \equiv \mathcal{T}(s, u)=\gamma(s)+u \varepsilon \dot{\gamma}(s),
\end{gathered}
$$

where $\varepsilon=\left(\begin{array}{cc}0 & -1 \\ 1 & 0\end{array}\right)$ is the rotation by $\pi / 2$, and hence $\varepsilon \dot{\gamma}(s)$ the inward normal. The map $\mathcal{T}$ is injective on

$$
\Omega_{0}^{e}:=\left\{(s, u) \in \Omega_{0} \mid s \in \mathbb{R}, 0 \leq u<w(s)\right\},
$$

with Jacobian $|\operatorname{det} \mathrm{D} \mathcal{T}|=1-u \kappa(s)$ uniformly bounded away from zero, for some sufficiently small positive, continuous width function $w(s)$. Due to condition (1.3) we may take it so that

$$
w(s) \geq c_{1}+c_{2}|s|
$$

for some $c_{1}, c_{2}>0$. The map (1.7) provides coordinates $(s, u)$ on the image $\Omega^{e}:=$ $\mathcal{T}\left(\Omega_{0}^{e}\right) \subset \Omega$ (Fig. 1, 2). Not all of $\Omega_{0}^{e}$ is essential for the sought identification, but only its tails near $s= \pm \infty$,

$$
\Omega_{0 \pm}^{e}:=\left\{(s, u) \in \Omega_{0}^{e} \mid \pm s>C\right\} .
$$

For large enough $C$ the tubular map is Euclidean if restricted to $\Omega_{0 \pm}^{e}$, since $\operatorname{supp} \ddot{\gamma}$ is compact. To make the dynamics of (1.4) and (1.6) comparable, we assume that

$$
A(x)=(-u, 0), \quad\left(x \in \Omega_{ \pm}^{e}\right)
$$

w.r.t. the Euclidean coordinates $(s, u)$ in $\Omega_{ \pm}^{e}:=\mathcal{T}\left(\Omega_{0 \pm}^{e}\right)$. This does not fix the potential $A$ outside of $\Omega_{-}^{e} \cup \Omega_{+}^{e}$ beyond the condition (1.5). Any residual gauge transformation $A \rightarrow A+\nabla \chi$ in $\Omega$ consistent with these requirements has $\chi(x)$ constant in $\Omega_{-}^{e} \cup \Omega_{+}^{e}$. In fact, $\chi(x)$ takes constant values $\chi_{ \pm}$separately on $\Omega_{ \pm}^{e}$, and

$$
\chi_{+}-\chi_{-}=\int_{-\infty}^{\infty} \nabla \chi(\gamma(s)) \cdot \dot{\gamma}(s) \mathrm{d} s=0 .
$$

The asymptotic Hilbert space $L^{2}\left(\Omega_{0}\right)$ is now mapped into $L^{2}(\Omega)$ by means of

$$
\begin{gathered}
J: L^{2}\left(\Omega_{0}\right) \rightarrow L^{2}(\Omega) \\
(J \psi)(x)=\left\{\begin{array}{cl}
j(u-w(s)) \psi(s, u), & \text { if } x=x(s, u) \in \Omega^{e}, \\
0, & \text { otherwise }
\end{array}\right.
\end{gathered}
$$

where $j \in C^{\infty}(\mathbb{R}), j \leq 1$ is such that

$$
j(u)= \begin{cases}1, & u \leq-2 w_{0}, \\ 0, & u \geq-w_{0},\end{cases}
$$

for some $w_{0}$. The purpose of the transition function $j$ is to make $J \psi$ as smooth as $\psi$. 
The first result establishes the usual properties of scattering.

Theorem 1 The wave operators

$$
\begin{aligned}
& W_{ \pm}: L^{2}\left(\Omega_{0}\right) \rightarrow L^{2}(\Omega) \\
& W_{ \pm}:=\underset{t \rightarrow \pm \infty}{\mathrm{s}-\lim _{t}} e^{i H t} J e^{-i H_{0} t}
\end{aligned}
$$

exist and are complete:

$$
\operatorname{Ran} W_{ \pm}=P_{\text {ac }}(H) \mathcal{H}
$$

Moreover, $W_{ \pm}$are isometries and do not depend on the choice of $w, j$ in the definition of $J$.

Remark 1 Under a residual gauge transformation the wave operators transform as

$$
W_{ \pm} \rightarrow e^{i\left(\chi_{ \pm}-\chi(x)\right)} W_{ \pm}
$$

implying by (1.10) that the scattering operator $W_{+}^{*} W_{-}$is invariant.

We next consider the limit where $\beta$ grows large while the energy, rescaled as in (1.4), is kept fixed. The limit of the scattering operator is thus best formulated in a scheme where edge states with fixed energy are displayed as being independent of $\beta$. The domain $\Omega_{0}$ is invariant under scaling

$$
(u, s) \rightarrow(\beta u, \beta s)
$$

and the Hamiltonian transforms as

$$
H_{0} \cong-\partial_{u}^{2}+\left(-i \partial_{s}+u\right)^{2}
$$

which shows that the spectrum of $H_{0}$ is independent of $\beta$. Let $\mathcal{H}_{T}:=L^{2}\left(\mathbb{R}_{+}, \mathrm{d} u\right)$ be the space of transverse wave functions, on which $-\partial_{u}^{2}$ acts with a Dirichlet boundary conditions at $u=0$. The translation invariance in $s$ of (1.14) calls for the (inverse) Fourier transform w.r.t. that variable

$$
\begin{gathered}
\mathcal{F}_{\beta}: \int^{\oplus} \mathcal{H}_{T} \mathrm{~d} k \cong L^{2}\left(\mathbb{R}, \mathcal{H}_{T}, \mathrm{~d} k\right) \rightarrow L^{2}\left(\mathbb{R}, \mathcal{H}_{T}, \mathrm{~d} s\right) \cong L^{2}\left(\Omega_{0}\right), \\
\psi=\int^{\oplus} \psi(k) \mathrm{d} k \mapsto \mathcal{F}_{\beta} \psi \\
\left(\mathcal{F}_{\beta} \psi\right)(s)=\underset{K \rightarrow \infty}{\lim . \operatorname{mon}} \frac{\beta^{1 / 2}}{(2 \pi)^{1 / 2}} \int_{-K}^{K} e^{i \beta k s} \mathcal{D}_{\beta} \psi(k) \mathrm{d} k
\end{gathered}
$$

where the scaling of $(s, u)$ has been incorporated for $u$ by means of

$$
\mathcal{D}_{\beta}: \mathcal{H}_{T} \rightarrow \mathcal{H}_{T}, \quad\left(\mathcal{D}_{\beta} \psi\right)(u)=\beta^{1 / 2} \psi(\beta u),
$$


and for $s$ explicitly in the integral. (It is, in a precise sense, a Bochner integral of $\mathcal{H}_{T}$-valued functions [1, Sect. 1.1, Sect. 1.8]). Upon displaying all variables, (1.15) reads

$$
\left(\mathcal{F}_{\beta} \psi\right)(s, u)=\frac{\beta}{(2 \pi)^{1 / 2}} \int_{-\infty}^{\infty} e^{i \beta k s} \psi(k, \beta u) \mathrm{d} k .
$$

Then

$$
\mathcal{F}_{\beta}^{-1} H_{0} \mathcal{F}_{\beta}=\widehat{H}_{0}:=\int^{\oplus} H_{0}(k) \mathrm{d} k, \quad H_{0}(k)=-\partial_{u}^{2}+(k+u)^{2} .
$$

The fiber $H_{0}(k)$, see [5], has simple, discrete spectrum $\left\{E_{n}(k)\right\}_{n \in \mathbb{N}}$ with projections denoted as $P_{n}(k)$. The energy curve $E_{n}(k)$, called the $n$-th Landau band, is a smooth function of $k$ increasing from $2 n+1$, the $n$-th Landau level, to $+\infty$ for $k \in(-\infty, \infty)$ with $E_{n}^{\prime}(k)>0$. The corresponding normalized eigenvectors $\psi_{n}(k)$ may be taken as smooth functions (in $\mathcal{H}_{T}$-norm) of $k$, though the choice is affected by the arbitrariness of their phase,

$$
\psi_{n}(k) \mapsto e^{i \lambda_{n}(k)} \psi_{n}(k) .
$$

They decay exponentially in $u$ (see Lemma 7).

In this scheme the scattering operator is

$$
S=\mathcal{F}_{\beta}^{-1} W_{+}^{*} W_{-} \mathcal{F}_{\beta}: \int^{\oplus} \mathcal{H}_{T} \mathrm{~d} k \rightarrow \int^{\oplus} \mathcal{H}_{T} \mathrm{~d} k
$$

It becomes independent of the magnetic field if large, and preserves the Landau bands:

Theorem 2 We have

$$
\operatorname{s-lim}_{\beta \rightarrow \infty} S=S_{\phi}
$$

with

$$
S_{\phi}=\int \sum_{n} e^{i \phi_{n}(k)} P_{n}(k) \mathrm{d} k .
$$

The scattering phases $\phi_{n}(k)$ are

$$
\phi_{n}(k)=-\frac{E_{n}^{(1)}(k)}{E_{n}^{\prime}(k)} \theta,
$$

where $\theta$ is the bending angle (1.2) and

$$
\begin{gathered}
E_{n}^{(1)}(k)=\left\langle\psi_{n}(k), H_{1}(k) \psi_{n}(k)\right\rangle, \\
H_{1}(k)=u^{3}+3 u^{2} k+2 u k^{2} .
\end{gathered}
$$


More precisely, if energies are restricted to any open interval $\Delta$ between Landau levels, as in (1.1), the limit (1.20) holds in norm: for any $\varepsilon>0$ there is $C_{\Delta, \varepsilon}$ such that

$$
\left\|\left(S-S_{\phi}\right) E_{\Delta}\left(\widehat{H}_{0}\right)\right\| \leq C_{\Delta, \varepsilon} \beta^{-1+\varepsilon} .
$$

We summarize the analysis which will later rigorously lead to this result and to its extension to higher orders in $\beta^{-1}$. In the limit considered, the cyclotron radius becomes small as compared to a typical radius of curvature. At any point along the boundary, the particle experiences a dynamics which differs only slightly from that along a straight boundary, provided the two dynamics are represented in a common Hilbert space. Once this is done, the system turns out to be mixed in the sense [10] that the Hamiltonian (1.4) corresponds to the quantization over a phase space, $\mathbb{R}^{2} \ni(s, k)$, of a classical symbol,

$$
H(s, k)=H_{0}(k)+\beta^{-1} \kappa(s) H_{1}(k)+\mathrm{O}\left(\beta^{-2}\right),
$$

taking values in the operators on a Hilbert space $\mathcal{H}_{T}$. Thus, $\beta^{-1} E_{n}^{(1)}(k)$ is the first order correction to the eigenvalue $E_{n}(k)$ under the (singular) perturbation $\kappa(s) H_{1}(k)$ of $H_{0}(k)$ due to the curvature of the boundary. Integrated over time it gives rise to the scattering phase $\phi_{n}$ in view of (1.2) and of the semiclassical expression $\beta(\mathrm{d} s / \mathrm{d} t)=E_{n}^{\prime}(k)$ for the velocity of the particle. Despite its relation to the geometry of $\Omega$, the origin of the scattering phase is not geometrical in the sense of Berry's, but appears to be dynamical. Corrections in all orders of $\beta^{-1}$ can be investigated systematically by means of Space Adiabatic Perturbation Theory [12], which applies to mixed systems as described above, of which (1.23) is an example.

The plan of the remaining sections is as follows. In the next section we give a heuristic interpretation of the edge states and of the scattering phases in terms of classical orbits bouncing at the boundary. Readers more interested in the proofs of Thms. 1 and 2 may proceed directly to Sects. 3 and 4, respectively. For instance, in Lemma 5 of the latter section (1.23) is stated in precise terms. Higher order corrections are discussed in Sect. 5, illustrating the use of Space Adiabatic Perturbation Theory. In particular it is shown there that the Landau bands are in fact preserved to arbitrary order in $\beta^{-1}$, while from (1.22) we may only conclude that they are preserved to order $\beta^{-1+\varepsilon}$.

\section{Classical trajectories and scattering phase}

We present a heuristic interpretation of the edge states and of the scattering phase $\phi_{n}(k)$ in terms of classical orbits. Related considerations are found in [8].

The Hamiltonian

$$
H_{0}=\left(\beta^{-1} p_{s}+\beta u\right)^{2}+\beta^{-2} p_{u}^{2},
$$

which is the classical counterpart to (1.6), has circular trajectories for which radius $r>0$ and velocity $v \in \mathbb{R}^{2}$ are in the fixed relation $r=|v| / 2$. Some of them bounce 
along the edge of the half-plane. Their shape may be parameterized in various ways: (i) By the ratio

$$
\frac{k}{r}=\cos \eta
$$

between the distance $k=\beta^{-2} p_{s}$ of the guiding center to $\partial \Omega_{0}$ (negative, if inside $\Omega_{0}$ ) and the radius $r$. This is also expressed through the angle $\eta$ between the boundary and the arc, see Fig. 3. (ii) By the ratio

$$
\frac{v_{\|}}{|v|}=\frac{\sin \eta}{\eta}
$$

between the average velocity $v_{\|}$along the edge and the (constant) velocity $|v|$ or, equivalently, between the length $2 r \sin \eta$ of the chord and $2 r \eta$ of the arc in Fig. 3 .
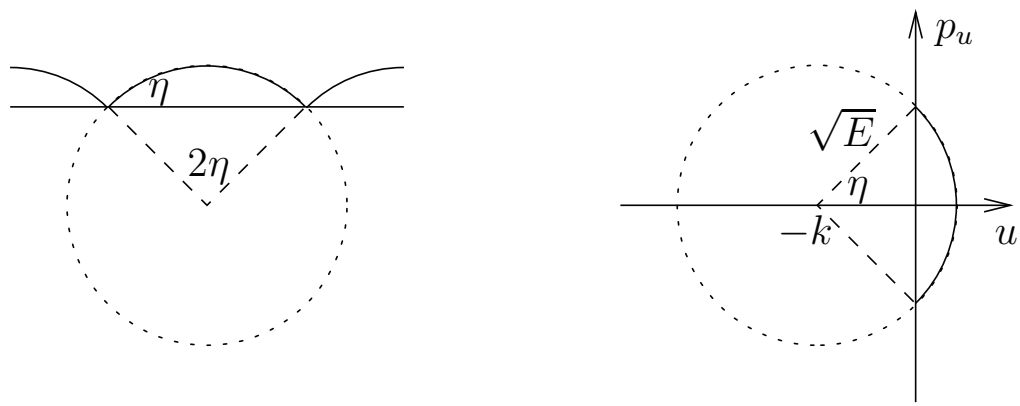

Figure 3. Left: a bouncing trajectory.

Figure 4. Right: the phase space of transversal motion.

We now turn to the quantum state $e^{i k s} \psi_{n}(k)$ for $\beta=1$, cf. (1.15). On the basis of (2.1) it may be associated, at least asymptotically for large $n$, with a classical trajectory of shape $\eta$ if

$$
k_{n}=\sqrt{E_{n}\left(k_{n}\right)} \cos \eta .
$$

The same conclusion is reached on the basis of (2.2) if $v_{\|}$is identified with the group velocity $E_{n}^{\prime}(k)$, as we presently explain. The phase space $\mathbb{R}_{+} \times \mathbb{R} \ni\left(u, p_{u}\right)$ underlying $H_{0}(k)$ is shown in Fig. 4, together with a trajectory of energy $(k+$ $u)^{2}+p_{u}^{2}=E$. Let $A(E, k)$ be the area of the cap inside this trajectory. The BohrSommerfeld condition, whose asymptotic validity we take for granted, states that $A\left(E_{n}(k), k\right)=2 \pi n,(n \in \mathbb{N})$, and derivation w.r.t. $k$ yields

$$
\frac{\partial A}{\partial E} E_{n}^{\prime}(k)+\frac{\partial A}{\partial k}=0 .
$$


Using that $-\partial A / \partial k$ is the length of the chord in Fig. 4, and $\frac{\partial A}{\partial \sqrt{E}}=2 \sqrt{E} \frac{\partial A}{\partial E}$ that of the arc we find

$$
\frac{v_{\|}}{|v|}=\frac{E_{n}^{\prime}(k)}{2 \sqrt{E_{n}(k)}}=\frac{\sin \eta}{\eta},
$$

provided $k=k_{n}$ is chosen as in (2.3). The energy is then $E_{n}\left(k_{n}\right) \propto n$ and the radius before the scaling (1.13) is given by $r_{n}^{2}=\beta^{-2} E_{n}\left(k_{n}\right)$.

Let

$$
G\left(s, s^{\prime} ; \kappa\right)=\int_{\gamma} p \cdot \mathrm{d} x
$$

be the (reduced) action along one of the two $\operatorname{arcs} \gamma$ of radius $r$ joining neighboring collision points $s$ and $s^{\prime}$ (provided they are close enough, so that the arcs exist). Since $\int_{\gamma} p \cdot \mathrm{d} x=\int_{\gamma} p_{s} \mathrm{~d} s+\int_{\gamma} p_{u} \mathrm{~d} u=\beta k\left(s^{\prime}-s\right)+A$, the Bohr-Sommerfeld condition ensures that the form of a quantum wave $e^{i \beta k s} \mathcal{D}_{\beta} \psi(k)$ is consistent with the semiclassical phase $e^{i G}$.

In light of this correspondence we shall discuss the motion along a curved boundary. The semiclassical limit, $n \gg 1$, and the limit of small curvature, $\kappa r_{n}$ $\ll 1$, are compatible as long as $1 \ll n \ll \beta^{2} \kappa^{-2}$, i.e., for large magnetic fields. We again first deal with the classical particle, whose incidence angle $\eta$ may now slightly change from hit to hit. Let now $s$ and $s^{\prime}$ in (2.5) denote the arc length coordinates of the two collision points along the boundary of curvature $\kappa(\cdot)$. With $p=\beta^{2}(v / 2+A)$ we obtain $[2]$

$$
G\left(s, s^{\prime} ; \kappa\right)=\beta^{2}(r \mathcal{L}-\mathcal{A}),
$$

where $\mathcal{L}$ is the length of the $\operatorname{arc} \gamma$ and $\mathcal{A}$ the area between the arc and the boundary $\partial \Omega$. In fact,

$$
\frac{1}{2} \int_{\gamma} v \cdot \mathrm{d} x=\frac{|v|}{2} \int_{\gamma} \frac{\mathrm{d} x}{\mathrm{~d} \sigma} \cdot \mathrm{d} x=r \int_{\gamma} \mathrm{d} \sigma,
$$

where $\sigma$ is the arc length along $\gamma$; and, by Stokes' theorem, $\int_{\gamma} A \cdot \mathrm{d} x=-\mathcal{A}$, because the arc is traversed clockwise and because of (1.5). We next consider an arc starting at $s$ with angle $\eta$ and look for the dependence of $s^{\prime}-s, \eta^{\prime}-\eta$ and $G\left(s, s^{\prime} ; \kappa\right)$ up to first order in a small curvature $\kappa$. Elementary considerations show that

$$
\begin{aligned}
& \delta\left(s^{\prime}-s\right) \approx-\kappa r^{2} \sin 2 \eta, \quad \delta\left(\eta^{\prime}-\eta\right) \approx 0, \\
& \delta \mathcal{L} \approx-2 \kappa r^{2} \sin \eta, \quad \delta \mathcal{A} \approx-\frac{4}{3} \kappa r^{3} \sin ^{3} \eta, \\
& \delta G\left(s, s^{\prime} ; \kappa\right) \approx-2 \beta^{2} r^{3} \kappa \sin \eta\left(1-\frac{2}{3} \sin ^{2} \eta\right),
\end{aligned}
$$

where $\kappa=\kappa(\tilde{s})$ for any $\tilde{s}$ between $s$ and $s^{\prime}$. We then take a number $m$ of hops $s_{i}=$ $s_{i}[\kappa],(i=0, \ldots m)$ sufficient to cover the bent $\operatorname{supp} \kappa$. Using $s_{i}-s_{i-1}=2 r \sin \eta$ 
for $\kappa \equiv 0$, we compute in the small curvature limit

$$
\begin{gathered}
\delta\left(s_{m}-s_{0}\right)=\sum_{i=1}^{m} \frac{\delta\left(s_{i}-s_{i-1}\right)}{s_{i}-s_{i-1}}\left(s_{i}-s_{i-1}\right) \approx-r \frac{\sin 2 \eta}{2 \sin \eta} \int_{-\infty}^{\infty} \kappa(s) \mathrm{d} s=-r \theta \cos \eta \\
\delta \sum_{i=1}^{m} G\left(s_{i-1}, s_{i} ; \kappa\right) \approx-\beta^{2} r^{2} \theta\left(1-\frac{2}{3} \sin ^{2} \eta\right) .
\end{gathered}
$$

An incoming quantum wave $e^{i \beta k s} \mathcal{D}_{\beta} \psi(k)$ should therefore gather an additional phase

$$
\phi_{n}(k)=-\beta k \delta\left(s_{m}-s_{0}\right)+\delta \sum_{i=1}^{m} G\left(s_{i-1}, s_{i} ; \kappa\right)
$$

as compared to one following a straight boundary of the same length. With (2.3) we find

$$
\phi_{n}\left(k_{n}\right)=\beta r_{n} \theta \sqrt{E_{n}\left(k_{n}\right)} \cos ^{2} \eta-\beta^{2} r_{n}^{2} \theta\left(1-\frac{2}{3} \sin ^{2} \eta\right)=-\frac{1}{3} \theta E_{n}\left(k_{n}\right) \sin ^{2} \eta .
$$

On the other hand, the phase $\phi_{n}\left(k_{n}\right)$ may be computed from (1.21). Since the trajectory in Fig. 4 is traversed at a uniform rate, expectations w.r.t. $\psi_{n}\left(k_{n}\right)$ reduce in the limit to integrations w.r.t. $(2 \eta)^{-1} \mathrm{~d} \alpha$, where a point on the arc is represented by its angle $\alpha \in[-\eta, \eta]$ as seen from the center of the circle. We rewrite $\sqrt{E} \cos \alpha=$ $k+u=: u^{\prime}$ and $u^{3}+3 u^{2} k+2 u k^{2}=u^{\prime}\left(u^{\prime 2}-k^{2}\right)$, use

$$
\frac{1}{2 \eta} \int_{-\eta}^{\eta} \cos \alpha \mathrm{d} \alpha=\frac{\sin \eta}{\eta}, \quad \frac{1}{2 \eta} \int_{-\eta}^{\eta} \cos ^{3} \alpha \mathrm{d} \alpha=\frac{\sin \eta}{\eta}\left(1-\frac{1}{3} \sin ^{2} \eta\right)
$$

and obtain

$$
\begin{gathered}
E_{n}^{(1)}(k) \approx E_{n}\left(k_{n}\right)^{3 / 2} \frac{\sin \eta}{\eta}\left(1-\frac{1}{3} \sin ^{2} \eta-\cos ^{2} \eta\right)=\frac{2}{3} E_{n}\left(k_{n}\right)^{3 / 2} \frac{\sin ^{3} \eta}{\eta}, \\
\phi_{n}\left(k_{n}\right) \approx-\frac{1}{3} \theta E_{n}\left(k_{n}\right) \sin ^{2} \eta,
\end{gathered}
$$

where we used (2.4) in the last step. The result is in agreement with (2.6).

\section{Existence and completeness of wave operators}

Existence and completeness of the wave operators $W_{ \pm}$follow in a rather standard way from propagation estimates for the dynamics $e^{-i H t}$ and $e^{-i H_{0} t}$.

Such an estimate is established in the second part of the following lemma. It depends on a Mourre estimate [7], which in turn rests on a geometric property discussed in the first part: 


\section{Lemma 1}

1. There is a function $\sigma \in C^{2}(\bar{\Omega})$ extending arc length from $\partial \Omega$ to $\Omega$, i.e., $\sigma(\gamma(s))=s$ for $s \in \mathbb{R}$, satisfying

$$
\left\|\partial_{i} \sigma\right\|_{\infty}<\infty, \quad\left\|\partial_{i} \partial_{j} \sigma\right\|_{\infty}<\infty .
$$

2. For any $\varepsilon>0, \alpha>1 / 2$ and $\Delta$ as in (1.1):

$$
\int_{-\infty}^{\infty}\left\|\langle\sigma\rangle^{-\alpha} e^{-i H t} E_{\Delta}(H) \psi\right\|^{2} \mathrm{~d} t \leq C_{\Delta, \alpha} \beta^{1+\varepsilon}\|\psi\|^{2}
$$

with $C_{\Delta, \alpha}$ independent of large enough $\beta$.

Proof. 1. On $\Omega_{0}$ we may choose the following extension of arc length:

$$
\sigma_{0}(s, u):=\frac{s}{w(s)}(w(s)-u) j_{\sigma}(u-w(s)),
$$

where $j_{\sigma}(u)$ is defined as in (1.12) with $w_{0}$ small enough and possibly smaller than $w_{0}$ used in the definition of $J$. It satisfies (3.1) and is supported on $\Omega_{0}^{e}$. We therefore obtain an extension of arc length $\sigma(x)$ from $\partial \Omega$ to $\Omega$ by transforming $\sigma_{0}$ under the tubular map:

$$
\sigma(x):= \begin{cases}\sigma_{0}(s, u) & \text { if } x=x(s, u) \in \Omega^{e} \\ 0 & \text { otherwise }\end{cases}
$$

$\sigma$ satisfies (3.1) because $\sigma_{0}$ is an extension of arc length on $\Omega_{0}, \sigma$ is supported on $\Omega^{e}$ and the inverse tubular map has bounded first and second derivatives on $\Omega^{e}$. The extension of $\sigma$ by zero to the complement of $\Omega^{e}$ is smooth by construction of $j_{\sigma}$.

2. To better display the dependence on $\beta$ of some of the bounds below we scale $\Omega$ to $\tilde{\Omega}=\beta \Omega$, so that $H \cong \tilde{H}$, where

$$
\tilde{H}=(-i \nabla-\tilde{A})^{2},
$$

on $L^{2}(\tilde{\Omega})$ with $\tilde{A}(x)=\beta A(x / \beta)$ corresponding to a unit magnetic field. The corresponding extension of arc length from part (1) is $\tilde{\sigma}(x)=\beta \sigma(x / \beta)$. We claim that for given $E \notin 2 \mathbb{N}+1$

$$
\begin{gathered}
\left\|[\tilde{H}, \tilde{\sigma}](\tilde{H}+i)^{-1}\right\| \leq C, \\
\|[[\tilde{H}, \tilde{\sigma}], \tilde{\sigma}]\| \leq C, \\
E_{\tilde{\Delta}}(\tilde{H}) i[\tilde{H}, \tilde{\sigma}] E_{\tilde{\Delta}}(\tilde{H}) \geq c E_{\tilde{\Delta}}(\tilde{H})
\end{gathered}
$$


with $C, c>0$ and an open interval $\tilde{\Delta} \ni E$, all independent of $\beta$ large. Indeed, (3.4), (3.5) follow from

$$
\begin{aligned}
i[\tilde{H}, \tilde{\sigma}]= & (-i \nabla-\tilde{A}) \cdot \nabla \tilde{\sigma}+\nabla \tilde{\sigma} \cdot(-i \nabla-\tilde{A}), \\
& i[i[\tilde{H}, \tilde{\sigma}], \tilde{\sigma}]=2(\nabla \tilde{\sigma})^{2},
\end{aligned}
$$

and (3.6) has been shown in connection with the proof of Thm. 3 in [7]. The bounds (3.4)-(3.6) now imply [9] for $\alpha>1 / 2$ :

$$
\int_{-\infty}^{\infty}\left\|\langle\tilde{\sigma}\rangle^{-\alpha} e^{-i \tilde{H} t} E_{\tilde{\Delta}}(\tilde{H}) \psi\right\|^{2} \mathrm{~d} t \leq C\|\psi\|^{2} .
$$

Undoing the unitary scale transformation, this amounts to:

$$
\beta^{-2 \alpha} \int_{-\infty}^{\infty}\left\|\left(\sigma^{2}+\beta^{-2}\right)^{-\alpha / 2} e^{-i H t} E_{\tilde{\Delta}}(H) \psi\right\|^{2} \mathrm{~d} t \leq C\|\psi\|^{2} .
$$

Using a covering argument for $\Delta$, this proves

$$
\int_{-\infty}^{\infty}\left\|\langle\sigma\rangle^{-\alpha} e^{-i H t} E_{\Delta}(H) \psi\right\|^{2} \mathrm{~d} t \leq C_{\Delta, \alpha} \beta^{2 \alpha}\|\psi\|^{2},
$$

for $\beta \geq 1$, which may be assumed without loss. For $\alpha \leq(1+\varepsilon) / 2$ the claim follows from $\beta^{2 \alpha} \leq \beta^{1+\varepsilon}$. It then extends to $\alpha>(1+\varepsilon) / 2$ because the l.h.s of (3.7) is decreasing in $\alpha$.

Remark 2 The bound (3.2) may be understood in simple terms. The velocity of a particle tangential to the boundary is $i[H, s]=\beta^{-1}\left(-i \beta^{-1} \nabla-\beta A(x)\right) \cdot \nabla s=$ $O\left(\beta^{-1}\right)$, assuming its energy $H$ lies in $\Delta$. It therefore takes the particle a time $O(\beta)$ to traverse a fixed piece of the boundary such as the bent. Eq. (3.2) is stating just this, up to a multiplicative error $O\left(\beta^{\varepsilon}\right)$.

We shall prove existence and completeness of the wave operators $W_{ \pm}$by local Kato smoothness. More precisely by [13, Thm. XIII.31] or, with more detail, by [14, Sect. 4.5, Thm. 1, Cor. 2, Rem. 3, Thm. 6] all of Thm. 1, except for the uniqueness statement, is implied by the following lemma:

\section{Lemma 2}

1. J maps $\mathcal{D}\left(H_{0}\right)$ into $\mathcal{D}(H)$. Moreover

$$
H J-J H_{0}=\sum_{i=1}^{2} A_{i}^{*} M_{i} A_{i}^{0},
$$

where $A_{i}^{(0)}$ are $H_{(0)}$-bounded and $H_{(0)}$-smooth on $\Delta$, and $M_{i}$ are bounded operators, $(i=1,2,(0)=0$ or its omission $)$. 
2 .

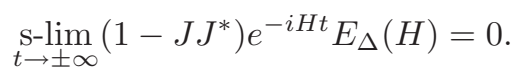

Proof. 1. For $C$ large enough, $\left|\sigma_{0}(s, u)\right|>C$ implies $j(u-w(s))=1$. In fact, if $j(u-w(s))<1$ we have $u-w(s)>-2 w_{0}$ and therefore, see Eq. (3.3),

$$
\left|\sigma_{0}(s, u)\right|=\frac{|s|}{w(s)}(w(s)-u) j_{\sigma}(u-w(s))
$$

is bounded by $2 w_{0} \sup _{s}|s| / w(s)$, which is finite by (1.8). By (3.10) we also see that $\left|\sigma_{0}(s, u)\right|>C$ implies that $|s|$ is large. These two implications, together with (1.9), show that $\left(H J-J H_{0}\right) F\left(\left|\sigma_{0}\right|>C\right)=0$, where $F(x \in A)$ is the characteristic function of the set $A$. Together with a similar relation for $\sigma$ instead of $\sigma_{0}$ we obtain

$$
H J-J H_{0}=\chi\left(H J-J H_{0}\right) \chi_{0},
$$

where $\chi_{(0)}=F\left(\left|\sigma_{(0)}\right| \leq C\right)$.

Eq. (3.11) may be written in the form (3.8) with

$$
\begin{aligned}
A_{1} & =\langle\sigma\rangle^{-\alpha}(H-i), \\
M_{1} & =\langle\sigma\rangle^{\alpha}(H+i)^{-1} \chi H J \chi_{0}\left\langle\sigma_{0}\right\rangle^{\alpha}, \\
A_{1}^{0} & =\left\langle\sigma_{0}\right\rangle^{-\alpha}, \\
A_{2} & =\langle\sigma\rangle^{-\alpha}, \\
M_{2} & =-\langle\sigma\rangle^{\alpha} \chi J H_{0} \chi_{0}\left(H_{0}+i\right)^{-1}\left\langle\sigma_{0}\right\rangle^{\alpha}, \\
A_{2}^{0} & =\left\langle\sigma_{0}\right\rangle^{-\alpha}\left(H_{0}+i\right) .
\end{aligned}
$$

The claimed properties about the $A_{i}^{(0)}$ hold true by (3.2) and we are left to show those of the $M_{i}^{(0)}$. Since $\chi\langle\sigma\rangle^{\alpha}, \chi_{0}\left\langle\sigma_{0}\right\rangle^{\alpha}$ (and $J$ ) are bounded, we need to show that

$$
H \chi(H+i)^{-1}\langle\sigma\rangle^{\alpha}=H \chi\left[\langle\sigma\rangle^{\alpha}(H+i)^{-1}+(H+i)^{-1}\left[\langle\sigma\rangle^{\alpha}, H\right](H+i)^{-1}\right]
$$

is, too (and similarly for the ' 0 '-version). Indeed, for $\alpha<1,\left[\langle\sigma\rangle^{\alpha}, H\right](H+i)^{-1}$ is bounded, cf. (3.4), and so is

$$
H f(H+i)^{-1}=H(H+i)^{-1}\left(f+[H, f](H+i)^{-1}\right)
$$

for $f=\chi\langle\sigma\rangle^{\alpha}$ or $f=\chi$.

2. Since $\left(1-J J^{*}\right)(1-\chi)=0$ and $\chi\langle\sigma\rangle^{\alpha}$ is bounded, we may show

$$
\lim _{t \rightarrow \pm \infty}\langle\sigma\rangle^{-\alpha} e^{-i H t} E_{\Delta}(H) \psi=0 .
$$

As a function of $t$, this state has bounded derivative and is square integrable in $t$, cf. (3.2). Hence the claim. 
It remains to show that $W_{ \pm}=W_{ \pm}(J)$ is independent of $j$ and $w$ in the construction (1.11) of $J$. We may choose $\tilde{\jmath}, \tilde{w}$ still satisfying the requirements (1.12), (1.8) and, moreover,

$$
\begin{gathered}
\operatorname{supp} \tilde{\jmath}(u-\tilde{w}(s)) \subset \Omega_{-}^{e} \cup \Omega_{+}^{e}, \\
\tilde{\jmath}(u-\tilde{w}(s)) j(u-w(s))=\tilde{\jmath}(u-\tilde{w}(s))
\end{gathered}
$$

for any two given choices $j=j_{i}, w=w_{i},(i=1,2)$. As for $\tilde{\jmath}$, this is achieved by taking a large enough $w_{0}$ in (1.12). To show $W_{ \pm}\left(J_{1}\right)=W_{ \pm}\left(J_{2}\right)$ it thus suffices to prove $W_{ \pm}(J)=W_{ \pm}(\tilde{J})$ for $J=J_{1}, J_{2}$. Since $(s, u)$ are Euclidean coordinates in $\Omega_{ \pm}^{e}$, Eqs. (3.12), (3.13) imply $\tilde{J} \tilde{J}^{*} J=\tilde{J}$ and therefore

$$
\begin{aligned}
& \mathrm{S}_{t \rightarrow \pm \infty}(J-\tilde{J}) e^{-i H_{0} t} E_{\Delta}\left(H_{0}\right)=\operatorname{s-lim}_{t \rightarrow \pm \infty}\left(1-\tilde{J} \tilde{J}^{*}\right) J e^{-i H_{0} t} E_{\Delta}\left(H_{0}\right)
\end{aligned}
$$

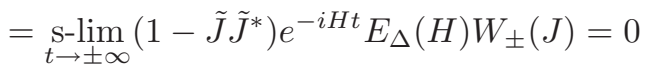

by (3.9), proving the claim.

\section{The scattering operator at large magnetic fields}

At large magnetic fields the scattering operator acquires a universal behavior, depending only on the bending angle, but independent of other geometric properties of the domain, as stated in Thm. 2. The estimate (1.22), from which the full statement of the theorem follows by density, will be established through an approximation to the evolution $e^{-i H t} \psi$ which is accurate at all times and not just near $t= \pm \infty$, as was the case in the previous section. To this end we choose an adapted gauge and interpret $H$ on $L^{2}(\Omega)$ as a perturbation of $H_{0}$ on $L^{2}\left(\Omega_{0}\right)$. This will require an identification of the two spaces which is more accurate than (1.11). Since these steps are intended for the limit $\beta \rightarrow \infty$, we will assume $\beta \geq 1$ throughout this section.

We begin with the choice of gauge, which is a deformation of Landau's.

Lemma 3 There is a smooth vector field on $\Omega$ with $\nabla \wedge A=1$ and (1.5), (1.9) whose pull-back on $\Omega_{0}^{e}$ under the tubular map, $A_{0}:=(\mathrm{D} \mathcal{T})^{t} A$, is

$$
A_{0}(s, u)=-\left(u-\frac{u^{2}}{2} \kappa(s), 0\right) .
$$

In the definition (1.19) of the scattering operator $S$ asymptotic states are represented as states in $\int^{\oplus} \mathcal{H}_{T} \mathrm{~d} k$ by means of $\mathcal{F}_{\beta}$, see (1.15). It is useful to make the band structure of $\widehat{H}_{0}$ explicit there. The range of $E_{\Delta}\left(H_{0}\right)$ then becomes isomorphic to the direct sum

$$
E_{\Delta}\left(H_{0}\right) \mathcal{H}_{0} \cong \bigoplus_{n \in \mathcal{B}} L^{2}\left(I_{n}, \mathrm{~d} k\right),
$$


where $I_{n}:=E_{n}^{-1}[\Delta]$ is bounded and $\mathcal{B}:=\left\{n \in \mathbb{N} \mid I_{n} \neq \emptyset\right\}$ is finite if $\Delta$ is as in Thm. 2. The isomorphism is established by the unitary

$$
\mathcal{U}: \bigoplus_{n \in \mathcal{B}} L^{2}\left(I_{n}, \mathrm{~d} k\right) \rightarrow E_{\Delta}\left(H_{0}\right) \mathcal{H}_{0}, \quad \mathcal{U}=\bigoplus_{n \in \mathcal{B}} U_{n}
$$

with

$$
U_{n}: L^{2}\left(I_{n}, \mathrm{~d} k\right) \rightarrow E_{\Delta}\left(H_{0}\right) \mathcal{H}_{0}, \quad U_{n} f:=\mathcal{F}_{\beta}\left(\psi_{n} f\right),
$$

i.e.,

$$
\left(U_{n} f\right)(s)=\frac{\beta^{1 / 2}}{(2 \pi)^{1 / 2}} \int_{I_{n}} e^{i \beta k s} \mathcal{D}_{\beta} \psi_{n}(k) f(k) \mathrm{d} k .
$$

The Hamiltonian for the $n$-th band, $U_{n}^{*} H_{0} U_{n}=: h_{n}$, is multiplication by $E_{n}(k)$. We define single band wave operators as

$$
\Omega_{ \pm}(n):=\underset{t \rightarrow \pm \infty}{s-\lim _{t}} e^{i H t} J U_{n} e^{-i h_{n} t}=W_{ \pm} U_{n},
$$

and corresponding scattering operators as

$$
\sigma_{n m}:=\Omega_{+}^{*}(n) \Omega_{-}(m) .
$$

At this point (1.22) reduces to

$$
\left\|\sigma_{n m}-\delta_{n m} e^{i \phi_{n}(k)}\right\|_{\mathcal{L}\left(L^{2}\left(I_{m}\right), L^{2}\left(I_{n}\right)\right)} \leq C_{\Delta, \varepsilon} \beta^{-1+\varepsilon} .
$$

An improved identification operator $\tilde{J}: L^{2}\left(\Omega_{0}\right) \rightarrow L^{2}(\Omega)$ is

$$
(\tilde{J} \psi)(x)=\left\{\begin{array}{cl}
j(u-w(s)) g(s, u)^{-1 / 4} \psi(s, u), & \text { if } x=x(s, u) \in \Omega^{e} \\
0, & \text { otherwise }
\end{array}\right.
$$

It is obtained as a modification of (1.11), where $g(s, u)^{1 / 2}=|\operatorname{det} \mathrm{D} \mathcal{T}|$ and $g \mathrm{~d} s \mathrm{~d} u$ is the Euclidean volume element $\mathrm{d} x_{1} \mathrm{~d} x_{2}$ in tubular coordinates. We take the parameter $w_{0}$ in (1.12) so that $3 w_{0}<\inf _{s} w(s)$. Then $j(u-w(s))=1$ for $u<w_{0}$ and $\tilde{J}$ acts as an isometry on states supported near $\partial \Omega_{0}$, which is where we expect edge states to be concentrated at all times.

The perturbation induced by the curvature of $\partial \Omega$ on the dynamics will be accounted for by a modification $\tilde{U}_{n}$ of $U_{n}$ in $(4.2)$, resp. $\tilde{\mathcal{J}}_{n}:=\tilde{J} \tilde{U}_{n}$ of $J U_{n}$ in (4.3):

$$
\begin{gathered}
\tilde{U}_{n}: L^{2}\left(I_{n}, \mathrm{~d} k\right) \rightarrow \mathcal{H}_{0} \\
\left(\tilde{U}_{n} f\right)(s):=\frac{\beta^{1 / 2}}{(2 \pi)^{1 / 2}} \int_{I_{n}} e^{i\left(\beta k s+\phi_{n}(s, k)\right)} \mathcal{D}_{\beta} \tilde{\psi}_{n}(s, k) f(k) \mathrm{d} k,
\end{gathered}
$$


where

$$
\begin{gathered}
\phi_{n}(s, k)=-\frac{E_{n}^{(1)}(k)}{E_{n}^{\prime}(k)} \int_{-\infty}^{s} \kappa\left(s^{\prime}\right) \mathrm{d} s^{\prime}, \\
\tilde{\psi}_{n}(s, k)=\psi_{n}(k)+\beta^{-1} \kappa(s) \tilde{\psi}_{n}^{(1)}(k), \\
\tilde{\psi}_{n}^{(1)}(k)=\psi_{n}^{(1)}(k)-\frac{E_{n}^{(1)}(k)}{E_{n}^{\prime}(k)}\left(\partial_{k} \psi_{n}\right)(k), \\
\psi_{n}^{(1)}(k)=-\left(H_{0}(k)-E_{n}(k)\right)^{-1}\left(1-P_{n}(k)\right) H_{1}(k) \psi_{n}(k) .
\end{gathered}
$$

It will be proved later that (4.7) yields a bounded map (4.6). Here we remark that $H_{1}(k) \psi_{n}(k)$ is well defined because $\psi_{n}(k)$ decays exponentially in $u$. A semiclassical interpretation of the above construction is in order. The evolution would adiabatically promote a particle from the asymptotic state $\psi_{n}(k)$ at $s=-\infty$ to the perturbed eigenstate $\psi_{n}^{[1]}(s, k)=\psi_{n}(k)+\beta^{-1} \kappa(s) \psi_{n}^{(1)}(k)$ of (1.23), if $k$ were an adiabatic invariant. It is only approximately so, since it changes by $\mathrm{d} k / \mathrm{d} t=\{H(s, k), k\} \approx-\beta^{-1} \dot{\kappa}(s) E_{n}^{(1)}(k)$ per unit time or, cumulatively w.r.t. arc length, by $\delta k(s)=-\beta^{-1} \kappa(s) E_{n}^{(1)}(k) / E_{n}^{\prime}(k)$. Therefore a more accurate state is $\psi_{n}^{[1]}(s, k+\delta k(s))=\tilde{\psi}_{n}(s, k)+\mathrm{O}\left(\beta^{-2}\right)$. This argument leaves open the possibility of an additional phase, $e^{i \beta^{-1} \gamma_{\mathrm{B}}(s, k)}$, determined by parallel transport, which however does not have to be included in (4.8). In fact, due to global gauge symmetry, only the derivative w.r.t. $s$ of the phase of the WKB ansatz (4.7) is determined by the Schrödinger equation, where it occurs in the combination $\beta^{-1} \partial_{s}$. The contribution of $\gamma_{\mathrm{B}}$ there is thus of order $\mathrm{O}\left(\beta^{-2}\right)$.

The main intermediate result of this section is that $\tilde{\mathcal{J}}_{n} e^{-i h_{n} t}$ is an accurate approximation of $e^{-i H t}$ at all times in the relevant energy range:

Proposition 1 For all $\varepsilon>0$ and $\Delta$ as in Thm. 1:

$$
\sup _{t \in \mathbb{R}}\left\|E_{\Delta}(H)\left(e^{-i H t} \tilde{\mathcal{J}}_{n}-\tilde{\mathcal{J}}_{n} e^{-i h_{n} t}\right)\right\|_{\mathcal{L}\left(L^{2}\left(I_{n}\right), L^{2}(\Omega)\right)} \leq C_{\Delta, \varepsilon} \beta^{-1+\varepsilon} .
$$

The implication of this result on the scattering operators $\sigma_{n m}$ can now be phrased conveniently in terms of Isozaki-Kitada wave operators $\tilde{\Omega}_{ \pm}(n)$ :

Proposition 2 The limits

$$
\tilde{\Omega}_{ \pm}(n)=\underset{t \rightarrow \pm \infty}{\mathrm{S}-\lim _{t}} e^{i H t} \tilde{\mathcal{J}}_{n} e^{-i h_{n} t}
$$

exist and equal

$$
\tilde{\Omega}_{-}(n)=\Omega_{-}(n), \quad \tilde{\Omega}_{+}(n)=\Omega_{+}(n) e^{i \phi_{n}(k)} .
$$

Moreover, for $\varepsilon>0$,

$$
\left\|\tilde{\Omega}_{+}^{*}(n) \tilde{\Omega}_{-}(m)-\delta_{n m}\right\| \leq C \beta^{-1+\varepsilon} .
$$


Since $\sigma_{n m}=e^{i \phi_{n}(k)} \tilde{\Omega}_{+}^{*}(n) \tilde{\Omega}_{-}(m)$, the proof of Eq. (4.4) and of Thm. 2 is complete, except for the proofs of Lemma 3 and Props. 1, 2 which we will give in the rest of this section.

Proof. (Lemma 3) We may first define $A(x)$ for $x \in \Omega_{e}$ so that (4.1) holds, i.e., in terms of forms $A=\left(\mathcal{T}^{*}\right)^{-1} A_{0}, A_{0}=-\left(u-\frac{u^{2}}{2} \kappa(s)\right) \mathrm{d} s$. We indeed have $\nabla \wedge A=1$ there, because

$$
\mathrm{d} A_{0}=-(1-u \kappa(s)) \mathrm{d} u \wedge \mathrm{d} s=g^{1 / 2} \mathrm{~d} s \wedge \mathrm{d} u,
$$

and thus $\mathrm{d} A=\left(\mathcal{T}^{*}\right)^{-1}\left(\mathrm{~d} A_{0}\right)=\mathrm{d} x_{1} \wedge \mathrm{d} x_{2}$, but also $\mathrm{d} A=(\nabla \wedge A) \mathrm{d} x_{1} \wedge \mathrm{d} x_{2}$. We also note that (1.5) holds, since $A(\gamma(s)) \cdot \dot{\gamma}(s)=\left.A_{0}\left(\partial_{s}\right)\right|_{u=0}=0$. The definition of $A$ can then be extended as follows to all of $\Omega$ : Starting from any field $\tilde{A}$ with $\nabla \wedge \tilde{A} \equiv 1$ on $\Omega$, there is $\chi(x)$ such that $A=\tilde{A}+\nabla \chi$ on $\Omega_{e}$. Now it suffices to extend the scalar function $\chi$ to $\Omega$.

Some of the further analysis is conveniently phrased in terms of pseudodifferential calculus, of which we shall need a simple version. We fix a band $n$ with momentum interval $I_{n}$ and drop the band index $n$ from all quantities throughout the remainder of this section. The symbols are defined on the phase space $\mathbb{R} \times I \ni(s, k)$ of a particle on the boundary $\partial \Omega$ and take values in some Banach space $X$, typically $X \subset \mathcal{H}_{T}$ :

$$
\mathcal{A}_{2}(X):=\left\{a \mid a(s, k) \in X,\|a\|_{\mathcal{A}_{2}(X)}^{2}:=\int \sup _{k \in I}\|a(s, k)\|_{X}^{2} \mathrm{~d} s<\infty\right\} .
$$

We abbreviate $\mathcal{A}_{2} \equiv \mathcal{A}_{2}\left(\mathcal{H}_{T}\right)$. If $X=\mathcal{D}(M)$ is the domain of some closed operator $M$ equipped with the graph norm $\|\cdot\|_{M}=\|\cdot\|_{\mathcal{H}_{T}}+\|M \cdot\|_{\mathcal{H}_{T}}$, we just write $\mathcal{A}_{2}(M) \equiv$ $\mathcal{A}_{2}(\mathcal{D}(M))$.

For a symbol $a \in \mathcal{A}_{2}(X)$, we define an operator by left-quantization

$$
\begin{gathered}
\operatorname{Op}(a): L^{2}(I) \rightarrow L^{2}(\mathbb{R}, X), \\
(\operatorname{Op}(a) f)(s):=\frac{\beta^{1 / 2}}{\sqrt{2 \pi}} \int_{I} e^{i \beta k s}\left(\mathcal{D}_{\beta} a\right)(s, k) f(k) \mathrm{d} k,
\end{gathered}
$$

where $\mathcal{D}_{\beta}$ is as in (1.16). The integral is a Bochner integral on $\mathcal{H}_{T}$ [1, Thm. 1.1.4]. It exists pointwise for each $s \in \mathbb{R}$ with $\sup _{k \in I}\|a(s, k)\|_{X}<\infty$, because $\mathcal{H}_{T}$ is separable and $\|f\|_{1} \leq|I|^{1 / 2}\|f\|_{2}$. Moreover, (4.14) defines a bounded operator $\mathrm{Op}(a): L^{2}(I) \rightarrow L^{2}(\mathbb{R}, X)$, because of

$$
\|\mathrm{Op}(a) f\| \leq \frac{(\beta|I|)^{1 / 2}}{\sqrt{2 \pi}}\|a\|_{\mathcal{A}_{2}(X)}\|f\|_{2} .
$$

We shall extend in two ways the class of symbols $a$ admissible in (4.14). First, that equation defines a bounded operator $L^{2}(I) \rightarrow L^{2}(\mathbb{R}, X)$ also if $a(s, k)$ tends 
to some asymptotes for some $a_{ \pm}(k)$ at large $s$, in the sense that

$$
\int_{0}^{ \pm \infty} \sup _{k \in I}\left\|a(s, k)-a_{ \pm}(k)\right\|_{X}^{2} \mathrm{~d} s<\infty, \quad \sup _{k \in I}\left\|a_{ \pm}(k)\right\|_{X}<\infty .
$$

We denote such symbols by $a \in \mathcal{A}(X)$. In fact, the integral is still defined pointwise as before; in the case that $a$ is independent of $s$ the result follows by the unitarity of the Fourier transform, and in general from $a(s, k)-\theta(s) a_{+}(k)-\theta(-s) a_{-}(k) \in$ $\mathcal{A}_{2}(X)$. (Further conditions for $\|\mathrm{Op}(a)\|<\infty$, which we shall not need, are given by the Calderón-Vaillancourt theorem [11].) Second, the notation (4.14) shall be used also when the symbol $a(s, k)$ is actually a polynomial in $\beta^{-1}, a(s, k)=$ $\sum_{j=0}^{\operatorname{deg} a} \beta^{-j} a_{j}(s, k)$, in which case $\|a(s, k)\|_{X}^{2}:=\sum_{j=0}^{\operatorname{deg} a}\left\|a_{j}(s, k)\right\|_{X}^{2}$. An example for both extensions is $a(s, k):=\tilde{\psi}(s, k) e^{i \phi(s, k)} \in \mathcal{A}\left(H_{0}(k)\right)$, for which $\mathrm{Op}(a)=\tilde{U}$. In particular (4.7) defines a bounded map, as claimed. Note that $\mathcal{D}\left(H_{0}(k)\right)$, see (1.17), is independent of $k$.

The following propagation estimate holds:

Lemma 4 Let $a \in \mathcal{A}_{2}$. Then

$$
\int_{-\infty}^{\infty}\left\|\mathrm{Op}(a) e^{-i h t} f\right\|^{2} \mathrm{~d} t \leq C \beta\|f\|^{2}
$$

where

$$
C=\int \sup _{k \in I} \frac{\|a(s, k)\|^{2}}{E^{\prime}(k)} \mathrm{d} s<\infty
$$

Moreover,

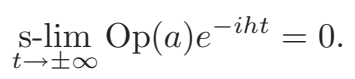

Proof. The integrand of the l.h.s. of (4.15) is

$$
\begin{array}{r}
\left\|\mathrm{Op}(a) e^{-i h t} f\right\|^{2}=\frac{\beta}{2 \pi} \int \mathrm{d} s \int_{I} \mathrm{~d} k_{2} \int_{I} \mathrm{~d} k_{1} e^{i \beta\left(k_{1}-k_{2}\right) s} e^{-i\left(E\left(k_{1}\right)-E\left(k_{2}\right)\right) t} \\
\times\left\langle a\left(s, k_{2}\right), a\left(s, k_{1}\right)\right\rangle \bar{f}\left(k_{2}\right) f\left(k_{1}\right),
\end{array}
$$

where we used that $\mathcal{D}_{\beta}$ is unitary. Formally, we may use

$$
\frac{1}{2 \pi} \int e^{-i\left(E\left(k_{1}\right)-E\left(k_{2}\right)\right) t} \mathrm{~d} t=\delta\left(E\left(k_{1}\right)-E\left(k_{2}\right)\right)=E^{\prime}\left(k_{1}\right)^{-1} \delta\left(k_{1}-k_{2}\right),
$$

because $k \mapsto E(k)$ is monotonous, so that (4.15) equals

$$
\beta \int \mathrm{d} s \int_{I} \mathrm{~d} k E^{\prime}(k)^{-1}\|a(s, k)\|^{2}\|f(k)\|^{2},
$$


from which the first claim follows. More carefully, we change variables $k_{i} \mapsto$ $E\left(k_{i}\right)=e_{i}, \mathrm{~d} k_{i}=E^{\prime}\left(k_{i}\right)^{-1} \mathrm{~d} e_{i}$ and extend the integrand by zero for $e_{i} \notin E^{-1}(I)$. Then (4.17) follows by Tonelli's theorem and Parseval's identity.

Eq. (4.16) follows from the fact that $\operatorname{Op}(a) e^{-i h t} f$ has bounded derivative in $t$ and is square integrable w.r.t. $t$.

Prop. 1 states that $\tilde{\mathcal{J}}=\tilde{J} \tilde{U}$ approximately intertwines between the dynamics $h$ on $L^{2}(I, \mathrm{~d} k)$ and $H$ on $\mathcal{H}$. Its proof will combine the intertwining properties of $\tilde{J}$ and of $\tilde{U}$, as discussed separately by the following two lemmas.

Lemma 5 Let

$$
H_{1}:=\beta^{-1}\left(2(\beta u) D_{s} \kappa D_{s}-\frac{1}{2}(\beta u)^{2}\left\{\kappa, D_{s}\right\}\right),
$$

where $D_{s}=-i \beta^{-1} \partial_{s}+\beta u$ and curly brackets denote the anticommutator. Then for any $1 / 2<\alpha \leq 1$ :

$$
\left(H \tilde{J}-\tilde{J}\left(H_{0}+H_{1}\right)\right) \tilde{U}=\langle\sigma\rangle^{-\alpha} R \mathrm{Op}(b),
$$

where $\|b\|_{\mathcal{A}_{2}} \leq C$ and $\|R\|_{\mathcal{L}\left(\mathcal{H}_{0}, \mathcal{H}\right)} \leq C_{\alpha} \beta^{-2}$.

Lemma 6 For any $\alpha>0$ we have:

$$
\left(H_{0}+H_{1}\right) \tilde{U}-\tilde{U} h=\left\langle\sigma_{0}\right\rangle^{-\alpha} R \mathrm{Op}(b),
$$

where $\|b\|_{\mathcal{A}_{2}} \leq C,\|R\|_{\mathcal{L}\left(\mathcal{H}_{0}\right)} \leq C_{\alpha} \beta^{-2}$ and $H_{1}$ as in Lemma 5 .

The first lemma states that on the image of $\tilde{U}$ the Hamiltonian $H$ is a perturbation of the half-plane Hamiltonian $H_{0}$. The leading part, $H_{1}$, of this perturbation is formally of order $\beta^{-1}$, because $\beta u$ and $D_{s}$ are of $O(1)$ on the image of $\tilde{U}$. Since the tangential velocity $i\left[H_{0}, s\right]=2 \beta^{-1} D_{s}$ is of order $\beta^{-1}$, the size of $H_{1}$ is thus inversely proportional to the time $\sim \beta$ (in units of the inverse cyclotron frequency) required by the particle to traverse the bent, i.e., $\operatorname{supp} \kappa$. The cumulated effect is thus of order 1, like the phase (4.8) which by the second lemma accounts for it to leading order. Subleading contributions occurring in either approximation are formally of order $\beta^{-2}$. They may be integrated in time and controlled by means of the propagation estimates in Lemmas 1, 4 .

Proof. (Proposition 1) Upon multiplication from the left by $e^{i H t}$ the quantity to be estimated is seen to be

$$
E_{\Delta}(H)\left(\tilde{\mathcal{J}}-e^{i H t} \tilde{\mathcal{J}} e^{-i h t}\right)=-i \int_{0}^{t} E_{\Delta}(H) e^{i H \tau}(H \tilde{\mathcal{J}}-\tilde{\mathcal{J}} h) e^{-i h \tau} \mathrm{d} \tau .
$$

We expand

$$
H \tilde{\mathcal{J}}-\tilde{\mathcal{J}} h=\left(H \tilde{J}-\tilde{J}\left(H_{0}+H_{1}\right)\right) \tilde{U}+\tilde{J}\left(\left(H_{0}+H_{1}\right) \tilde{U}-\tilde{U} h\right),
$$


and insert the two terms on the r.h.s. into (4.20). We use the general fact that

$$
\|T\|=\sup \left\{\left|\left\langle\varphi_{2}, T \varphi_{1}\right\rangle\right| \mid \varphi_{i} \in \mathcal{H}_{i},\left\|\varphi_{i}\right\|=1,(i=1,2)\right\}
$$

for operators $T: \mathcal{H}_{1} \rightarrow \mathcal{H}_{2}$ between Hilbert spaces, and apply the estimates (4.18), (4.19) on the two contributions respectively. For the second term we also use $\tilde{J}\left\langle\sigma_{0}\right\rangle^{-\alpha}=\langle\sigma\rangle^{-\alpha} \tilde{J}$. Together with (3.2), (4.15), we see that the two contributions are bounded in norm by a constant times $\beta^{-2} \cdot \beta^{(1+\varepsilon) / 2} \cdot \beta^{1 / 2}=\beta^{-1+\varepsilon / 2}$.

The proofs of Lemmas 5, 6 are postponed till after that of Proposition 2.

Proof. (Proposition 2) Let $F(s \in A)$ be the characteristic function of the set $A \subset \mathbb{R}$. We claim that for any $a \in \mathbb{R}$

$$
\operatorname{sim}_{t \rightarrow-\infty} F(s \geq-a) \tilde{U} e^{-i h t}=0,
$$

and similarly for $U$ instead of $\tilde{U}$, as well as for $F(s \leq a)$ and $t \rightarrow+\infty$. It will be enough to prove (4.21) when acting on $f \in C_{0}^{\infty}(I)$.

We then have

$$
\left(\tilde{U} e^{-i h t} f\right)(s)=\frac{\beta^{1 / 2}}{(2 \pi)^{1 / 2}} \int_{I} e^{i(\beta k s-E(k) t+\phi(s, k))} \mathcal{D}_{\beta} \tilde{\psi}(s, k) f(k) \mathrm{d} k
$$

with

$$
\begin{aligned}
\frac{\partial}{\partial k}(\beta k s-E(k) t+\phi(s, k)) & =\beta s-E^{\prime}(k) t-\frac{\mathrm{d}}{\mathrm{d} k}\left(\frac{E^{(1)}(k)}{E^{\prime}(k)}\right) \int_{-\infty}^{s} \kappa\left(s^{\prime}\right) \mathrm{d} s^{\prime} \\
& \geq 1+\beta|s+a|+\delta|t|
\end{aligned}
$$

for some $\delta>0$, all $s \geq-a$ and $-t$ large enough. We may pretend that $\tilde{\psi}(s, k)$ is replaced by $\psi(k)$, as the difference is dealt with by (4.16). Since the latter amplitude is independent of $s$, the usual non-stationary phase method (e.g., [13, Thm. XI.14 and Corollary]) may be applied. We obtain (without keeping track of the dependence of constants on $\beta$ )

$$
\left\|\left(\tilde{U} e^{-i h t} f\right)(s)\right\|_{\mathcal{H}_{T}} \leq C_{l}(1+|s+a|+|t|)^{-l}, \quad(l \in \mathbb{N}, s \geq-a)
$$

where we also used that $\psi(k) \in C^{\infty}\left(I, \mathcal{H}_{T}\right)$. As a result,

$$
\left\|F(s \geq-a) \tilde{U} e^{-i h t} f\right\|^{2} \leq C_{l}^{\prime}(1+|t|)^{-2 l+1}
$$

for $-t$ large enough, proving (4.21). As the estimate (4.22) also holds with $\phi(s, k)$ omitted or replaced by $\phi(k)=\phi(s=\infty, k)$, the result applies to $U$ and $U e^{i \phi(k)}$ as well. 
We maintain that (4.21) implies

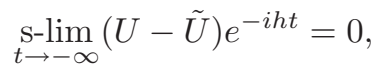

$$
\begin{aligned}
& \underset{t \rightarrow+\infty}{\operatorname{sim}}\left(U e^{i \phi(k)}-\tilde{U}\right) e^{-i h t}=0,
\end{aligned}
$$

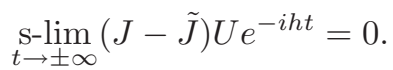

Indeed, if $-a<\operatorname{supp} \kappa$, and hence $e^{i \phi(s, k)}=1$ as well as $\tilde{\psi}(s, k)=\psi(k)$ for $s<-a$, then

$$
U-\tilde{U}=F(s \geq-a)(U-\tilde{U})
$$

and (4.23) follows from (4.21). Eq. (4.24) is shown similarly by using $\phi(s, k)=\phi(k)$ for $s>\operatorname{supp} \kappa$. Eq. (4.25) follows from $J-\tilde{J}=(J-\tilde{J}) F(|s| \leq a)$, since $g(s, u)=1$ for $(s, u) \in \Omega_{0}^{e},|s| \geq a$. Now (4.10), (4.11) are immediate. They follow from the existence of the wave operators (4.3), i.e., $\Omega_{ \pm}(n)=\mathrm{s}-\lim _{t \rightarrow \pm \infty} e^{i H t} J U e^{-i h t}$, by means of (4.25) and of (4.23), resp. (4.24).

Finally, we prove (4.12). Here it is necessary to introduce the band labels again. By the intertwining property of $\tilde{\Omega}_{ \pm}(n)$ between $H$ and $h_{n}$ we have

$$
\begin{aligned}
& \left\langle g, \tilde{\Omega}_{+}^{*}(n) \tilde{\Omega}_{-}(m) f\right\rangle=\left\langle\tilde{\Omega}_{+}(n) g, E_{\Delta}(H) \tilde{\Omega}_{-}(m) f\right\rangle \\
& =\lim _{t \rightarrow \infty}\left\langle e^{i H t} \tilde{\mathcal{J}}_{n} e^{-i h_{n} t} g, E_{\Delta}(H) e^{-i H t} \tilde{\mathcal{J}}_{m} e^{i h_{m} t} f\right\rangle \\
& =\lim _{t \rightarrow \infty}\left\langle\tilde{\mathcal{J}}_{n} e^{-i h_{n} t} g, E_{\Delta}(H) e^{-2 i H t} \tilde{\mathcal{J}}_{m} e^{i h_{m} t} f\right\rangle .
\end{aligned}
$$

By Proposition 1 this inner product equals, up to a function of $t$ bounded by $C \beta^{-1+\varepsilon}\|g\|\|f\|$, the expression

$$
\begin{aligned}
& \left\langle\tilde{\mathcal{J}}_{n} e^{-i h_{n} t} g, E_{\Delta}(H) \tilde{\mathcal{J}}_{m} e^{-i h_{m} t} f\right\rangle=\left\langle e^{i H t} \tilde{\mathcal{J}}_{n} e^{-i h_{n} t} g, E_{\Delta}(H) e^{i H t} \tilde{\mathcal{J}}_{m} e^{-i h_{m} t} f\right\rangle \\
& \stackrel{t \rightarrow+\infty}{\rightarrow}\left\langle\tilde{\Omega}_{+}(n) g, \tilde{\Omega}_{+}(m) f\right\rangle=\left\langle e^{i \phi_{n}(k)} g, \Omega_{+}^{*}(n) \Omega_{+}(m) e^{i \phi_{m}(k)} f\right\rangle=\delta_{n m}\langle g, f\rangle,
\end{aligned}
$$

proving (4.12). In the last line we used $\Omega_{+}^{*}(n) \Omega_{+}(m)=\delta_{n m} \operatorname{Id}_{L^{2}\left(I_{m}\right)}$. This follows from $W_{+}^{*} W_{+}=\operatorname{Id}_{\mathcal{H}_{0}}$ and $U_{n}^{*} U_{m}=\delta_{n m} \operatorname{Id}_{L^{2}\left(I_{m}\right)}$.

It remains to prove Lemmas 5,6 .

An element of pseudodifferential calculus [11] is the symbolic product. We will need the product of an operator valued symbol $h \in \mathcal{A}\left(\mathcal{L}\left(X, \mathcal{H}_{T}\right)\right)$ with a vector valued one, $a \in \mathcal{A}(X)$, which in the case that $h(s, k)$ is a polynomial in $k$ is defined as

$$
(h \bigsqcup a)(s, k):=\sum_{l=0}^{\operatorname{deg} h} \frac{\beta^{-l}}{i^{l} l !}\left(\partial_{k}^{l} h\right)(s, k) \cdot\left(\partial_{s}^{l} a\right)(s, k),
$$

since the sum is then finite. In applications of this product it is understood that $a \in C_{s}^{\operatorname{deg} h}(\mathcal{A}(X))$, where $a \in C_{s}^{l}(\mathcal{A}(X))$ means $\partial_{s}^{j} a \in \mathcal{A}(X), 0 \leq j \leq l$. 
Proof. (Lemma 6) Set $a(s, k):=\tilde{\psi}(s, k) e^{i \phi(s, k)} \in \mathcal{A}\left(H_{0}(k)\right)$. Then by Lemma 8 ,

$$
H_{0} \tilde{U}=H_{0} \mathrm{Op}(a)=\mathrm{Op}\left(H_{0}(k) \natural a\right),
$$

where $H_{0}(k)$ is given in (1.17). The operator $H_{1}$ may be written as

$$
\begin{aligned}
H_{1}=\beta^{-1} \kappa(s)\left[2(\beta u)\left(-i \beta^{-1} \partial_{s}\right)^{2}+\right. & \left.3(\beta u)^{2}\left(-i \beta^{-1} \partial_{s}\right)+(\beta u)^{3}\right] \\
& -i \beta^{-2} \dot{\kappa}(s)\left[2(\beta u)\left(-i \beta^{-1} \partial_{s}\right)+3 / 2(\beta u)^{2}\right] .
\end{aligned}
$$

According to Lemma 7 we have $k^{l} \downarrow a \in \mathcal{A}\left(e^{\lambda u}\right),(l=0,1,2)$, for some $\lambda>0$. Therefore, by Lemma 8:

$$
H_{1} \tilde{U}=\beta^{-1} \mathrm{Op}\left(\kappa(s)\left(H_{1} \curvearrowleft a\right)\right)+\beta^{-2} \mathrm{Op}\left(\dot{\kappa}(s)\left(H_{2} \curvearrowleft a\right)\right),
$$

where

$$
H_{1}(k)=2 u k^{2}+3 u^{2} k+u^{3}, \quad H_{2}(k)=-i\left(2 u k+(3 / 2) u^{2}\right) .
$$

By evaluating the expression

$$
H_{0} \curvearrowleft a=\sum_{l=0}^{2} \frac{\beta^{-l}}{i^{l} l !}\left(\partial_{k}^{l} H_{0}\right)(k) \cdot\left(\partial_{s}^{l} a\right)(s, k)
$$

we find:

$$
H_{0} \curvearrowleft a=\tilde{a}_{00}+\beta^{-1} a_{01}+\beta^{-2} \tilde{a}_{02},
$$

where $\tilde{a}_{00}=E(k) \tilde{\psi}(s, k) e^{i \phi(s, k)}, a_{01}=-\kappa(s) H_{1}(k) \psi(k) e^{i \phi(s, k)}$ and $\tilde{a}_{02} \in \mathcal{A}_{2}$ (coefficients with a tilde may themselves contain higher order terms in $\beta^{-1}$ ). The derivation is as follows: The r.h.s. of (4.27) equals

$$
H_{0} \natural a=\left[H_{0}(k) \tilde{\psi}(s, k)+\beta^{-1}\left(\partial_{s} \phi(s, k)\right) H_{0}^{\prime}(k) \psi(k)\right] e^{i \phi(s, k)}+O\left(\beta^{-2}\right) .
$$

The first contribution equals

$$
H_{0}(k) \tilde{\psi}(s, k)=E(k) \tilde{\psi}(s, k)+\beta^{-1} \kappa(s)\left[\frac{E^{(1)}(k)}{E^{\prime}(k)} H_{0}^{\prime}(k)-H_{1}(k)\right] \psi(k),
$$

which follows because (4.9) provides the eigenvector at first order,

$$
\left(\left(H_{0}(k)-E(k)\right)\left(\psi(k)+\beta^{-1} \kappa(s) \psi^{(1)}(k)\right)=\beta^{-1} \kappa(s)\left(E^{(1)}(k)-H_{1}(k)\right) \psi(k),\right.
$$

and from taking the derivative of $\left(H_{0}(k)-E(k)\right) \psi(k)=0$,

$$
\left(H_{0}(k)-E(k)\right)\left(\partial_{k} \psi\right)(k)=E^{\prime}(k) \psi(k)-H_{0}^{\prime}(k) \psi(k) .
$$

Since $\partial_{s} \phi=-\left(E^{(1)} / E^{\prime}\right) \kappa$ we see that the second term within the square brackets of (4.28) is canceled inside the first one. Hence

$$
H_{0} \curvearrowleft a=\left[E(k) \tilde{\psi}(s, k)-\beta^{-1} \kappa(s) H_{1}(k) \psi(k)\right] e^{i \phi(s, k)}+O\left(\beta^{-2}\right)
$$

accounting for $\tilde{a}_{00}$ and $a_{01}$. 
$H_{1} \natural a$ and $H_{2} \natural a$ are evaluated straightforwardly:

$$
\begin{aligned}
& \beta^{-1} \kappa(s) H_{1} \natural a=\beta^{-1} a_{11}+\beta^{-2} \tilde{a}_{12}, \\
& \beta^{-2} \dot{\kappa}(s) H_{2} \natural a=\beta^{-2} \tilde{a}_{22},
\end{aligned}
$$

where $a_{11}(s, k)=\kappa(s) H_{1}(k) \psi(k) e^{i \phi(s, k)}$ and $\tilde{a}_{i j}(s, k) \in \mathcal{A}_{2}$.

Collecting our expansions we get

$$
\left(H_{0}+H_{1}\right) \tilde{U}=\operatorname{Op}\left(\tilde{a}_{00}\right)+\beta^{-1} \mathrm{Op}\left(a_{01}+a_{11}\right)+\beta^{-2} \mathrm{Op}(b),
$$

where $b \in \mathcal{A}_{2}$. Since $\operatorname{Op}\left(\tilde{a}_{00}\right)=\tilde{U} h$ and $a_{01}+a_{11}=0$ we conclude that

$$
\left(H_{0}+H_{1}\right) \tilde{U}-\tilde{U} h=\beta^{-2} \mathrm{Op}(b) .
$$

We may extract a smooth characteristic function $\chi$ of $\operatorname{supp} \kappa$ from $\mathrm{Op}(b)$. Then (4.19) follows with $R=\beta^{-2}\left\langle\sigma_{0}\right\rangle^{\alpha} \chi(s)$.

Inspection of the proof shows that derivatives up to $\ddot{\kappa}(s)$ were assumed bounded. This holds true if $\gamma \in C^{4}$, as assumed in the Introduction.

Proof. (Lemma 5) We begin by factorizing (4.18) as

$$
\left(H \tilde{J}-\tilde{J}\left(H_{0}+H_{1}\right)\right) \tilde{U}=\langle\sigma\rangle^{-\alpha} \cdot Q \cdot\langle s\rangle^{-1} R_{\lambda}\langle s\rangle \cdot\langle s\rangle^{-1} e^{\lambda \beta u}\left(H_{0}+i\right) \tilde{U},
$$

where $\lambda>0$ is picked small, $R_{\lambda}=e^{\lambda \beta u}\left(H_{0}+i\right)^{-1} e^{-\lambda \beta u}$, and

$$
Q=\langle\sigma\rangle^{\alpha}\left(H \tilde{J}-\tilde{J}\left(H_{0}+H_{1}\right)\right)\langle s\rangle e^{-\lambda \beta u} .
$$

The claim will be established through

$$
\begin{gathered}
\|Q\|_{\mathcal{L}\left(\mathcal{D}\left(H_{0}\right), \mathcal{H}_{0}\right)} \leq C \beta^{-2}, \\
\left\|\langle s\rangle^{-1} R_{\lambda}\langle s\rangle\right\|_{\mathcal{L}\left(\mathcal{H}_{0}, \mathcal{D}\left(H_{0}\right)\right)} \leq C, \\
\langle s\rangle^{-1} e^{\lambda \beta u}\left(H_{0}+i\right) \tilde{U}=\operatorname{Op}(b), \quad\|b\|_{\mathcal{A}_{2}} \leq C,
\end{gathered}
$$

where $\|\cdot\|_{\mathcal{A}_{2}}$ is the norm in (4.13).

Indeed, (4.31) follows from

$$
\langle s\rangle^{-1} R_{\lambda}\langle s\rangle=R_{\lambda}-\langle s\rangle^{-1} R_{\lambda}\left[H_{0},\langle s\rangle\right] R_{\lambda}
$$

and $R_{\lambda} \in \mathcal{L}\left(\mathcal{H}_{0}, \mathcal{D}\left(H_{0}\right)\right), \sup _{\beta \geq 1}\left\|R_{\lambda}\right\|_{\mathcal{L}\left(\mathcal{H}_{0}, \mathcal{D}\left(H_{0}\right)\right)}<\infty$.

Turning to (4.32), we recall that by $(4.26)\left(H_{0}+i\right) \tilde{U}=\mathrm{Op}(a)$ with $a \in \mathcal{A}$ (though $a \notin \mathcal{A}_{2}$, cf. $\left.\tilde{a}_{00}\right)$. For $\lambda$ small enough we have $a \in \mathcal{A}\left(e^{\lambda u}\right)$ by Lemma 7 . We conclude that $b=\langle s\rangle^{-1} e^{\lambda u} a \in \mathcal{A}_{2}$. 
In order to show (4.30), we have to determine how $H \tilde{J}$ acts. For $\varphi \in C_{0}^{\infty}\left(\bar{\Omega}_{0}\right)$, $\left.\varphi\right|_{\partial \Omega_{0}}=0$ a direct computation yields:

$$
(H \tilde{J} \varphi)(x)= \begin{cases}\left(g^{-1 / 4} \tilde{H} j \varphi\right)(s, u), & x=x(s, u) \in \Omega^{e}, \\ 0 & \text { otherwise, }\end{cases}
$$

where $j=j(u-w(s))$ and $\tilde{H}$ is the differential operator on $\Omega_{0}^{e}$

$$
\begin{gathered}
\tilde{H}=g^{1 / 4}\left(g^{-1 / 2} \tilde{D}_{i} g^{1 / 2} g^{i j} \tilde{D}_{j}\right) g^{-1 / 4}, \\
\tilde{D}_{s}=-i \beta^{-1} \partial_{s}+\beta u-\frac{\beta u^{2}}{2} \kappa(s), \quad \tilde{D}_{u}=-i \beta^{-1} \partial_{u}, \\
g(s, u)=(1-u \kappa(s))^{2}, \quad g^{i j}=\left(\begin{array}{cc}
g^{-1} & 0 \\
0 & 1
\end{array}\right) .
\end{gathered}
$$

In (4.33) summation over $i, j=s, u$ is understood. The expression inside the brackets is the Laplace-Beltrami operator in tubular coordinates on $\Omega_{0}^{e}$ associated to the covariant derivative $-i \beta^{-1} \nabla-\beta A$ on $\Omega^{e}$. Here we used Lemma 3 .

Eq. (4.33) has been rearranged in [6, Thm. 3.1] as $\tilde{H}=T+\beta^{-2} V$ with

$$
\begin{gathered}
T=\tilde{D}_{s} g^{-1} \tilde{D}_{s}-\beta^{-2} \partial_{u}^{2} \\
V(s, u)=\frac{1}{2} g^{-3 / 2} \frac{\partial^{2} \sqrt{g}}{\partial s^{2}}-\frac{5}{4} g^{-2}\left(\frac{\partial \sqrt{g}}{\partial s}\right)^{2}-\frac{1}{4} g^{-1}\left(\frac{\partial \sqrt{g}}{\partial u}\right)^{2} .
\end{gathered}
$$

Thus,

$$
(H \tilde{J} \varphi)(x)= \begin{cases}\left(g^{-1 / 4}\left(T+\beta^{-2} V\right) j \varphi\right)(s, u), & x=x(s, u) \in \Omega^{e}, \\ 0 & \text { otherwise. }\end{cases}
$$

States of the form

$$
\tilde{\psi}(x)= \begin{cases}g^{-1 / 4}(s, u) \psi(s, u), & x=x(s, u) \in \Omega^{e}, \\ 0 & \text { otherwise }\end{cases}
$$

have norm $\|\tilde{\psi}\|^{2}=\int_{\Omega_{0}^{e}}|\psi(s, u)|^{2} \mathrm{~d} s \mathrm{~d} u \leq\|\psi\|^{2}$. Since $V(s, u)$ is bounded on $\Omega_{0}^{e}$ and of compact support in $s$, its contribution to $Q$ is seen to satisfy (4.30). As for $T$, we write

$$
T=\left(D_{s} g^{-1} D_{s}-\beta^{-2} \partial_{u}^{2}\right)-\frac{\beta^{-1}}{2}\left\{(\beta u)^{2} \kappa g^{-1}, D_{s}\right\}+\frac{\beta^{-2}}{4}(\beta u \kappa)^{2} g^{-1} .
$$

We next Taylor expand $g^{-1}$ to first, resp. zeroth order in $u$ in the first two terms,

$$
\begin{aligned}
g^{-1} & =1+2 u \kappa+g^{-1}(3-2 u \kappa)(u \kappa)^{2}, \\
& =1+g^{-1}(2-u \kappa)(u \kappa),
\end{aligned}
$$


and lump the remainders together with the last term of (4.36). These three remainder contributions to (4.35) have compact support in $s$ and are bounded by $\beta^{-2}$ (in the graph norm of $H_{0}$ ) after multiplication by $e^{-\lambda \beta u}$, as in (4.29). They thus comply with (4.30). The expanded terms in (4.36) are

$$
D_{s}^{2}-\beta^{-2} \partial_{u}^{2}+\beta^{-1}\left(2(\beta u) D_{s} \kappa D_{s}-\frac{1}{2}(\beta u)^{2}\left\{\kappa, D_{s}\right\}\right)=H_{0}+H_{1} .
$$

All this means that in proving (4.30) we may now pretend that $H \tilde{J}$ is given by (4.35) with $T+\beta^{-2} V$ replaced by $H_{0}+H_{1}$. This is to be compared with

$$
\left(\tilde{J}\left(H_{0}+H_{1}\right) \varphi\right)(x)= \begin{cases}\left(g^{-1 / 4} j\left(H_{0}+H_{1}\right) \varphi\right)(s, u), & x=x(s, u) \in \Omega^{e}, \\ 0 & \text { otherwise. }\end{cases}
$$

The resulting commutator is computed as

$$
\left[H_{0}+H_{1}, j\right]=-i \beta^{-1}\left\{D_{i}, \partial_{i} j\right\}-i \beta^{-2}\left(2 \beta u\left\{\kappa \partial_{s} j, D_{s}\right\}-(\beta u)^{2} \kappa \partial_{s} j\right) .
$$

Its contribution to (4.29) is estimated by a constant times $e^{-\lambda \beta w_{0} / 4}$ thanks to the choice of $w_{0}$ made after (4.5). Therefore (4.30) is proved.

\section{Higher order approximations: Space Adiabatic Perturbation Theory}

In this section we outline how higher order approximations of the scattering operator can be achieved by means of Space Adiabatic Perturbation Theory [12]. We aim at the following generalization of Proposition 2:

Proposition 3 For all $l \geq 1$ there exists an identification $\tilde{\mathcal{J}}_{n}: L^{2}\left(I_{n}\right) \rightarrow L^{2}(\Omega)$ and a phase function $\phi_{n}^{(l-1)}(k)=\sum_{j=0}^{l-1} \beta^{-j} \phi_{j}(k)$ such that the limits

$$
\tilde{\Omega}_{ \pm}(n)=\operatorname{s-lim}_{t \rightarrow \pm \infty} e^{i H t} \tilde{\mathcal{J}}_{n} e^{-i h_{n} t}
$$

exist and equal

$$
\tilde{\Omega}_{-}(n)=\Omega_{-}(n), \quad \tilde{\Omega}_{+}(n)=\Omega_{+}(n) e^{i \phi_{n}^{(l-1)}(k)} .
$$

Moreover, for $\varepsilon>0$,

$$
\left\|\tilde{\Omega}_{+}^{*}(n) \tilde{\Omega}_{-}(m)-\delta_{n m}\right\| \leq C \beta^{-l+\varepsilon} .
$$

The scattering operator is then approximated by $S_{\phi}^{(l)}:=\int{ }^{\oplus} \sum_{n} e^{i \phi_{n}^{(l-1)}(k)} P_{n}(k) \mathrm{d} k$ up to errors of order $O\left(\beta^{-l+\varepsilon}\right)$,

$$
\left\|\left(S-S_{\phi}^{(l)}\right) E_{\Delta}\left(\widehat{H}_{0}\right)\right\| \leq C_{\Delta, \varepsilon} \beta^{-l+\varepsilon} .
$$

Proposition 3 also establishes that interband scattering is in fact strongly suppressed, i.e., suppressed to arbitrary order in $\beta^{-1}$ : 


\section{Corollary 1}

$$
\forall n \neq m: \quad\left\|\sigma_{n m}\right\|_{\mathcal{L}\left(L^{2}\left(I_{m}\right), L^{2}\left(I_{n}\right)\right)}=O\left(\beta^{-\infty}\right) .
$$

As before the improved identifications are decomposed as $\tilde{\mathcal{J}}_{n}=\tilde{J} \tilde{U}_{n}$, where $\tilde{J}$ is the operator (4.5). The proof of Proposition 2 carries over to that of Proposition 3 if $\tilde{U}_{n}$ satisfies the following requirements:

$$
\begin{aligned}
& \operatorname{sim}_{t \rightarrow-\infty}\left(U_{n}-\tilde{U}_{n}\right) e^{-i h_{n} t}=0,
\end{aligned}
$$

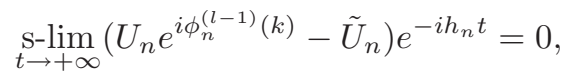

$$
\begin{aligned}
& \sup _{t \in \mathbb{R}}\left\|E_{\Delta}(H)\left(e^{-i H t} \tilde{\mathcal{J}}_{n}-\tilde{\mathcal{J}}_{n} e^{-i h_{n} t}\right)\right\|_{\mathcal{L}\left(L^{2}\left(I_{n}\right), L^{2}(\Omega)\right)} \leq C_{\Delta, \varepsilon} \beta^{-l+\varepsilon} .
\end{aligned}
$$

Complete proofs of these statements will be given in [3]. Here we shall only present a heuristic derivation.

The identification $\tilde{\mathcal{J}}_{n}$ is composed of the two intertwiners $\tilde{J}$ and $\tilde{U}_{n} . \tilde{J}$ is essentially just a coordinate transformation from Euclidean coordinates on $\Omega^{e}$ to tubular coordinates on $\Omega_{0}^{e}$. Its task is to intertwine the Hamiltonian $H$ on $L^{2}(\Omega)$ with some effective Hamiltonian $\tilde{H}$ that acts on $L^{2}\left(\Omega_{0}\right)$ and represents the effects of the boundary by an appropriate dynamic potential. In Section $4 \tilde{H}=H_{0}+H_{1}$ served this purpose. For higher order approximations we have to improve $\tilde{H} . \tilde{J}$ intertwines more accurately between $H$ and the $l$-th order semiclassical approximation $\tilde{H}^{(l)}$ of $T+\beta^{-2} V$, where $T$ and $V$ are the kinetic and potential energies of $(4.34)$,

$$
H \tilde{J}-\tilde{J} \tilde{H}^{(l)}=O\left(\beta^{-(l+1)}\right) .
$$

By semiclassical approximation we mean that $\hat{H}^{(l)}:=\mathcal{D}_{\beta}^{-1} \tilde{H}^{(l)} \mathcal{D}_{\beta}$ can be written as the Weyl quantization of some symbol $H^{(l)}(s, k)=\sum_{j=0}^{L} \beta^{-j} H_{j}(s, k)$, (here $L=$ $l+2)$, where the symbols $H_{j}(s, k)$ do not depend on $\beta^{-1}$ anymore. $\tilde{H}^{(l)}$ is obtained from $T+\beta^{-2} V$ by pushing the Taylor expansion of $g^{-1}$ to appropriate higher orders of $u$ in the expressions (4.34) and (4.36) for $V$ and $T$ respectively. Upon rescaling to magnetic length each additional order $u$ contributes an additional order $\beta^{-1}$. This leaves to $\tilde{U}_{n}$ the task of accurately intertwining $\tilde{H}^{(l)}$ and $h_{n}$ :

$$
\tilde{H}^{(l)} \tilde{U}_{n}-\tilde{U}_{n} h_{n}=O\left(\beta^{-(l+1)}\right) .
$$

In the last section we invoked the adiabatic nature of the evolution in order to motivate our construction of the approximate intertwiner $\tilde{U}_{n}$. This property can be exploited more systematically by means of Space Adiabatic Perturbation Theory (SAPT) [12], which allows to construct intertwiners $\tilde{U}_{n}$ at all orders $l$. Such approximations have to be sufficiently explicit of course in order to be of use.

SAPT applies to mixed quantum systems whose Hamiltonian $\hat{H}$ is the quantization of some operator valued semiclassical symbol $H(z) \asymp \sum_{l=0}^{\infty} \varepsilon^{l} H_{l}(z)$ w.r.t. some small parameter $\varepsilon . z \in \mathbb{R}^{2 d}$ is a phase space variable and the Hilbert space 
is $L^{2}\left(\mathbb{R}^{d}, \mathcal{H}_{f}\right)$, where $\mathcal{H}_{f}$ is some other separable Hilbert space, called the space of fast degrees of freedom. In our case $d=1, z=(s, k), \varepsilon=\beta^{-1}$ and $\mathcal{H}_{f}=\mathcal{H}_{T}$. The role of the Hamiltonian $\hat{H}$ is played by $\hat{H}^{(l)}=\mathcal{D}_{\beta}^{-1} \tilde{H}^{(l)} \mathcal{D}_{\beta}$.

SAPT associates to each spectral band $\sigma(z)$ of the principal symbol $H_{0}(z)$ that is separated by a gap from the rest of the spectrum an effective Hamiltonian $\hat{\mathfrak{h}}$ that acts on a fixed Hilbert space $L^{2}\left(\mathbb{R}^{d}, \mathcal{K}_{r}\right)$, where $\mathcal{K}_{r}$ can be any Hilbert space isomorphic to $\pi_{0}(z) \mathcal{H}_{f}$ for any $z \in \mathbb{R}^{2 d}$. Here $\pi_{0}(z)$ is the spectral projection of $H_{0}(z)$ that corresponds to $\sigma(z)$. The effective Hamiltonian is the quantization of a semiclassical symbol $\mathfrak{h} \asymp \sum_{l=0}^{\infty} \varepsilon^{l} \mathfrak{h}_{l}$. The symbol can be computed explicitly using a recursive scheme. In our case the spectral band $\sigma(z)$ is identified with one of the Landau bands $E_{n}(k) . \pi_{0}(s, k) \equiv P_{n}(k)$ is one dimensional and therefore $\mathcal{K}_{r} \equiv \mathbb{C}$. $\mathfrak{h}$ is a $\mathbb{C}$-valued symbol. The main results of SAPT imply the following statement:

The effective Hamiltonian is approximately intertwined with $\hat{H}$ by an isometry

$$
\mathfrak{J}: L^{2}\left(\mathbb{R}^{d}, \mathcal{K}_{r}\right) \rightarrow L^{2}\left(\mathbb{R}^{d}, \mathcal{H}_{f}\right),
$$

s.t.

$$
\hat{H} \mathfrak{J}-\mathfrak{J} \hat{\mathfrak{h}}=O\left(\varepsilon^{\infty}\right)
$$

Approximations of $\mathfrak{J}$ can be computed explicitly in terms of its Weyl-symbol to any finite order in $\varepsilon$.

In our context the physical meaning of this statement is that at any order in $\beta^{-1}$ the motion of the particle along the boundary is effectively one-dimensional at large $\beta$. It is described to a very good approximation by an effective Hamiltonian without a transverse degree of freedom. The effective Hamiltonian embodies all effects of the transverse degree of freedom on the longitudinal one.

In view of (5.4) and (5.5) we express $\tilde{U}_{n}$ as the composition of three distinct intertwiners,

$$
\tilde{U}_{n}=\mathcal{D}_{\beta} \mathfrak{J}^{(l)} \mathfrak{w}^{(l)} .
$$

The scaling $\mathcal{D}_{\beta}$ intertwines $\tilde{H}^{(l)}$ and $\hat{H}^{(l)} \cdot \mathfrak{J}^{(l)}$ is an approximation of $\mathfrak{J}$ up to order $O\left(\beta^{-(l+1)}\right)$ and approximately intertwines $\hat{H}^{(l)}$ and $\hat{\mathfrak{h}}$. $\mathfrak{w}^{(l)}$ then has to intertwine $\hat{\mathfrak{h}}$ and $h_{n}$ up to order $O\left(\beta^{-(l+1)}\right)$. This can be accomplished by standard WKB methods. A formal exact intertwiner $\mathfrak{w}$ between $\hat{\mathfrak{h}}$ and $h_{n}$ is constructed using generalized eigenfunctions of $\hat{\mathfrak{h}}$ :

$$
(\mathfrak{w} f)(s)=\frac{\beta^{1 / 2}}{\sqrt{2 \pi}} \int_{I_{n}} B(s, k) e^{i \beta S(s, k)} \mathrm{d} k,
$$

where formally

$$
\begin{gathered}
\hat{\mathfrak{h}} B(s, k) e^{i \beta S(s, k)}=E_{n}(k) \cdot B(s, k) e^{i \beta S(s, k)}, \\
\lim _{s \rightarrow-\infty}\left(B(s, k) e^{i \beta S(s, k)}-e^{i \beta k s}\right)=0 .
\end{gathered}
$$

$B(s, k)$ and $S(s, k)$ are assumed to be real functions. Their respective WKB approximations yield the approximate intertwiner $\mathfrak{w}^{(l)}$. The scattering phase results 
from $S(s, k)$ by taking the limit

$$
\phi^{(l-1)}(k)=\lim _{s \rightarrow+\infty} \beta(S(s, k)-k s) .
$$

The above derivation is rather formal. Neither did we show that (5.1), (5.2) hold nor is it clear from the discussion that the error terms are integrable in time along the evolution which is necessary to prove (5.3). The latter seems plausible, however, because we saw in the last section that the correction to the first order approximation of $\tilde{U}_{n}$ is integrable along the evolution.

In fact a closer look at the technical assumptions made in [12] about the symbol $H_{0}(z)$ reveals that our symbol $H_{0}(k)$ fails to comply with some of them. Apart from taking values in the unbounded operators, which causes minor technical complications, it violates the so-called gap condition. This is a condition on the growth of the symbol $H_{0}(k)$ with respect to $k$ relative to the growth of the respective gaps between the Landau bands. The condition is used in the general setting of [12] in order to control the global behavior of the various symbols w.r.t. the phase space variable $z$. The formal algebraic relationships between them, which are inherently local, are not affected. As is pointed out in [12, Sect. 4.5] this does not mean that SAPT is not applicable. It just means that suitable modifications to the general formalism have to be made in order to cover the special case at hand.

From the above heuristics we extract the following recipe for computing the scattering phase up to and including order $O\left(\beta^{-(l-1)}\right)$ :

1. Compute $\hat{H}^{(l)}$.

2. Compute $\mathfrak{h}$, the symbol of the effective Hamiltonian, that corresponds to $\hat{H}^{(l)}$ up to and including order $O\left(\beta^{-l}\right)$ using the formalism of [12].

3. Compute the scattering phase $\phi_{n}^{(l-1)}(k)$ from a sufficiently accurate WKB approximation of the generalized eigenfunction of $\hat{\mathfrak{h}}$ with eigenvalue $E_{n}(k)$.

Following these steps we find for $\phi^{(1)}(k)=\phi_{0}(k)+\beta^{-1} \phi_{1}(k)$, dropping the band index $n$ again,

$$
\begin{aligned}
& \phi_{0}(k)=-\frac{E^{(1)}(k)}{E^{\prime}(k)} \int_{-\infty}^{\infty} \kappa\left(s^{\prime}\right) \mathrm{d} s^{\prime}, \\
& \phi_{1}(k)=\left[\frac{1}{2}\left(\partial_{k}\left(\frac{E^{(1)}(k)}{E^{\prime}(k)}\right)^{2}+\left(\frac{E^{(1)}(k)}{E^{\prime}(k)}\right)^{2} \cdot \frac{E^{\prime \prime}(k)}{E^{\prime}(k)}\right)\right. \\
&\left.-\frac{E^{(1 ; 2)}(k)+E^{(2)}(k)}{E^{\prime}(k)}\right] \cdot \int_{-\infty}^{\infty} \kappa^{2}\left(s^{\prime}\right) \mathrm{d} s^{\prime},
\end{aligned}
$$

where $E^{(1 ; 2)}(k):=\left\langle\psi(k), H_{2}(k) \psi(k)\right\rangle, H_{2}(k):=u^{4}+1 / 4\left(u^{2}-1\right)+4 u^{3} k+3 u^{2} k^{2}$ is the second order Weyl-symbol of $\hat{H}^{(2)}$ with $\kappa^{2}$ stripped from it, and $E^{(2)}(k)$ is 
the second order correction to the eigenvalue $E(k)$ due to the perturbation $H_{1}(k)$,

$$
E^{(2)}(k):=\sum_{\substack{m=0 \\ m \neq n}}^{\infty} \frac{\left|\left\langle\psi_{n}(k), H_{1}(k) \psi_{m}(k)\right\rangle\right|^{2}}{E_{n}(k)-E_{m}(k)},
$$

where $n$ is the fixed band index.

The phase was computed from the WKB-ansatz (5.6) w.r.t. an effective Hamiltonian whose principal, first and second order symbols are

$$
\begin{gathered}
\mathfrak{h}_{0}=E(k), \quad \mathfrak{h}_{1}(s, k)=\kappa(s) E^{(1)}(k), \\
\mathfrak{h}_{2}(s, k)=\kappa^{2}(s)\left(E^{(1 ; 2)}(k)+E^{(2)}(k)\right)-E^{\prime}(k) \partial_{s} \gamma_{B}(s, k)-E^{\prime}(k) \partial_{s} \gamma_{R W}(s, k),
\end{gathered}
$$

where $\gamma_{\mathrm{B}}(s, k):=\kappa(s) \frac{E^{(1)}(k)}{E^{\prime}(k)} \operatorname{Im}\left\langle\psi(k), \partial_{k} \psi(k)\right\rangle$ and $\gamma_{\mathrm{RW}}(s, k):=-\gamma_{\mathrm{B}}(s, k)+$ $\frac{\kappa(s)}{E^{\prime}(k)} \operatorname{Im}\left\langle H_{1}(k) \psi(k), \partial_{k} \psi(k)\right\rangle$ are the leading order Berry and Rammal-Wilkinson phases (up to a factor $\beta^{-1}$ ) of the mixed system described by $\hat{H}^{(2)}$ [10]. The action function $S(s, k)$ has to satisfy the Hamilton Jacobi equation up to order $\beta^{-3}$,

$$
\mathfrak{h}\left(s, \partial_{s} S(s, k)\right)-E(k)=O\left(\beta^{-3}\right),
$$

while $B(s, k)$ has to satisfy the amplitude transport equation [10]

$$
\partial_{s}\left[B(s, k)^{2} \cdot \frac{\partial \mathfrak{h}}{\partial k}\left(s, \partial_{s} S\right)\right]=O\left(\beta^{-2}\right) .
$$

The Hamilton Jacobi equation (5.7) is solved by

$$
\begin{aligned}
& S(s, k)=k s-\beta^{-1} \frac{E^{(1)}(k)}{E^{\prime}(k)} \int_{-\infty}^{s} \kappa\left(s^{\prime}\right) \mathrm{d} s^{\prime}+\beta^{-2}\left(\gamma_{B}(s, k)+\gamma_{R W}(s, k)\right) \\
& +\beta^{-2}\left[\frac{1}{2}\left(\partial_{k}\left(\frac{E^{(1)}(k)}{E^{\prime}(k)}\right)^{2}+\left(\frac{E^{(1)}(k)}{E^{\prime}(k)}\right)^{2} \cdot \frac{E^{\prime \prime}(k)}{E^{\prime}(k)}\right)-\frac{E^{(1 ; 2)}(k)+E^{(2)}(k)}{E^{\prime}(k)}\right] \\
& \times \int_{-\infty}^{s} \kappa^{2}\left(s^{\prime}\right) \mathrm{d} s^{\prime},
\end{aligned}
$$

which leads to the stated scattering phase.

It is possible to modify the formalism of SAPT as developed in [12] and tailor it to our needs so that we can express $\tilde{U}_{n}$ at any order as an operator $\operatorname{Op}(a)$. The symbol $a$ is explicit enough as to enable us to prove (5.1), (5.2), (5.3) rigorously by essentially the same methods as in the last section. Moreover, the same formalism allows for a straightforward recursive computation of the scattering phase without reference to the concept of generalized eigenfunctions and their WKB approximations. A detailed account of this approach will be presented in [3]. 


\section{Appendix}

\subsection{Exponential decay}

Lemma 7 Let $I \subset \mathbb{R}$ be a compact interval. For each $n \in \mathbb{N}$ there exists $C<\infty$ such that for small $\lambda \geq 0$ and all $k \in I$ :

1.

$$
\left\|e^{\lambda u} \psi_{n}(k)\right\|_{\mathcal{H}_{T}} \leq C
$$

2 .

$$
\left\|e^{\lambda u}\left(\partial_{k} \psi_{n}+\left\langle\partial_{k} \psi_{n}, \psi_{n}\right\rangle \psi_{n}\right)\right\|_{\mathcal{H}_{T}} \leq C
$$

3.

$$
\left\|e^{\lambda u} \psi_{n}^{(1)}(k)\right\|_{\mathcal{H}_{T}} \leq C
$$

where $\psi_{n}^{(1)}(k)=-\left(H_{0}(k)-E(k)\right)^{-1}\left(1-P_{n}(k)\right) H_{1}(k) \psi_{n}(k)$ as in $(4.9)$.

Proof. The following norms refer to $\mathcal{H}_{T}$ or $\mathcal{L}\left(\mathcal{H}_{T}\right)$, as appropriate. By a covering argument we may assume $I$ to be small as needed.

1. Let $\Gamma \subset \rho(H(k)),(k \in I)$, be compact. We have

$$
\sup _{z \in \Gamma, k \in I}\left\|e^{\lambda u}\left(H_{0}(k)-z\right)^{-1} e^{-\lambda u}\right\|<\infty
$$

for small $\lambda$. In fact,

$$
e^{\lambda u} H_{0}(k) e^{-\lambda u}=H_{0}(k)+2 \lambda \partial_{u}-\lambda^{2}
$$

differs from $H_{0}(k)$ by a relatively bounded perturbation, and is thus an analytic family for small $\lambda$. Its resolvent, which appears within norms in (6.3), is therefore bounded. This implies

$$
\left\|e^{\lambda u} P_{n}(k) e^{-\lambda u}\right\|<\infty,
$$

where $\Gamma$ in

$$
P_{n}(k)=\frac{-1}{2 \pi i} \oint_{\Gamma}\left(H_{0}(k)-z\right)^{-1} \mathrm{~d} z
$$

is a contour encircling $E_{n}(k),(k \in I)$, counterclockwise. Since (6.4) equals $\left\|e^{\lambda u} \psi_{n}(k)\right\|\left\|e^{-\lambda u} \psi_{n}(k)\right\| \geq c\left\|e^{\lambda u} \psi_{n}(k)\right\|$ with $c>0$, Eq. (6.1) follows.

2. We have $\partial_{k} P_{n}(k)=\left|\partial_{k} \psi_{n}\right\rangle\left\langle\psi_{n}|+| \psi_{n}\right\rangle\left\langle\partial_{k} \psi_{n}\right|$, so that (6.2) equals

$$
\left\|e^{\lambda u}\left(\partial_{k} P_{n}\right) \psi_{n}(k)\right\| \leq\left\|e^{\lambda u}\left(\partial_{k} P_{n}\right) e^{-\lambda u}\right\|\left\|e^{\lambda u} \psi_{n}(k)\right\| .
$$

The claim then follows from (6.1),

$$
\partial_{k} P_{n}(k)=\frac{1}{2 \pi i} \oint_{\Gamma}\left(H_{0}(k)-z\right)^{-1}\left(\partial_{k} H_{0}(k)\right)\left(H_{0}(k)-z\right)^{-1} \mathrm{~d} z,
$$

as well as from (6.3) and $e^{\lambda u}\left(\partial_{k} H_{0}\right) e^{-\lambda u}=\partial_{k} H_{0}$. 
3. Finally, the last statement follows similarly from the representation of the reduced resolvent

$$
\left(H_{0}(k)-E(k)\right)^{-1}\left(1-P_{n}(k)\right)=\frac{1}{2 \pi i} \oint_{\Gamma}\left(H_{0}(k)-z\right)^{-1}\left(z-E_{n}(k)\right)^{-1} \mathrm{~d} z .
$$

\subsection{Left-Quantization}

Lemma 8 1. Let $T$ be some closed operator with $\mathcal{D}(T) \subset \mathcal{H}_{T}$. If a $\in \mathcal{A}(T)$ then $(\mathrm{Op}(a) f)(s) \in \mathcal{D}(T) \forall s \in \mathbb{R}$ and

$$
(1 \otimes T) \operatorname{Op}(a) f=\operatorname{Op}\left(\mathcal{D}_{\beta}^{-1} T \mathcal{D}_{\beta} a\right) f .
$$

2. Let $a \in C_{s}^{l}(\mathcal{A}(X))$ for some $l \in \mathbb{N}$, where $X \subset \mathcal{H}_{T}$ with $\|\cdot\|_{\mathcal{H}_{T}} \leq C\|\cdot\|_{X}$. Then $k^{l} \curvearrowleft a \in \mathcal{A}(X)$ and $(\mathrm{Op}(a) f)(s)$ is l-times differentiable in $s$ with

$$
\left(-i \beta^{-1} \partial_{s}\right)^{l}(\operatorname{Op}(a) f)=\operatorname{Op}\left(k^{l}\lfloor a) f .\right.
$$

3. Let $a \in C_{s}^{2}\left(\mathcal{A}\left(H_{0}(k)\right)\right)$. Then $H_{0} \natural a \in \mathcal{A}\left(\mathcal{H}_{T}\right)$, and $\mathrm{Op}(a) f \in \mathcal{D}\left(H_{0}\right)$ with

$$
H_{0} \mathrm{Op}(a)=\mathrm{Op}\left(H_{0} \curvearrowleft a\right) .
$$

Proof. 1. is an immediate consequence of [1, Proposition 1.1.7].

2. The integrand $e^{i \beta k s} \mathcal{D}_{\beta} a(s, k) f(k)$ of $(\mathrm{Op}(a) f)(s)$ is $l$-times differentiable in $s$ because $a \in C_{s}^{l}(\mathcal{A}(X))$. An application of the Leibniz rule yields

$$
\begin{aligned}
\left(-i \beta^{-1} \partial_{s}\right)^{l}\left(e^{i \beta k s} \mathcal{D}_{\beta} a(s, k) f(k)\right)=\mathcal{D}_{\beta} & \left(\sum_{m=0}^{l} \frac{\beta^{-m}}{i^{m} m !}\left(\partial_{k}^{m} k^{l}\right)\left(\partial_{s}^{m} a\right)(s, k)\right) \\
& \times e^{i \beta k s} f(k)=\mathcal{D}_{\beta}\left(k^{l} \natural a\right)(s, k) e^{i \beta k s} f(k) .
\end{aligned}
$$

Clearly $k^{l} \downarrow a \in \mathcal{A}(X)$. In particular $\left\|\partial_{s}^{l}\left(e^{i \beta k s} \mathcal{D}_{\beta} a(s, k) f(k)\right)\right\|_{X} \leq C|f(k)|$. Since $f(k) \in L^{1}(I, X)$, the claim follows by dominated convergence.

3. We have $\mathcal{D}\left(H_{0}(k)\right) \subset \mathcal{D}\left(D_{u}^{2}\right) \cap \mathcal{D}\left(u^{2}\right)$. Then, by 1 , we have that

$$
D_{u}^{2} \mathrm{Op}(a) f=\mathrm{Op}\left(-\partial_{u}^{2} a\right) f .
$$

Moreover 2. implies

$$
D_{s}^{2} \mathrm{Op}(a) f=\mathrm{Op}\left(k^{2}\lfloor a) f+2(\beta u) \mathrm{Op}(k \llbracket a) f+(\beta u)^{2} \mathrm{Op}(a) f,\right.
$$

where $k^{l} \downarrow a \in \mathcal{A}\left(H_{0}(k)\right),(l=0,1,2)$. Since $\mathcal{A}\left(H_{0}(k)\right) \subset \mathcal{A}\left(u^{2}\right)$, 1. implies that the r.h.s. of the last equation equals

$$
\mathrm{Op}\left(\left(k^{2} \natural a\right)+2 u(k \curvearrowleft a)+u^{2} a\right) f=\mathrm{Op}\left((k+u)^{2} \natural a\right) f .
$$

Combining (6.5), (6.6) we find

$$
H_{0} \mathrm{Op}(a) f=\left(D_{u}^{2}+D_{s}^{2}\right) \mathrm{Op}(a) f=\mathrm{Op}\left(-\partial_{u}^{2} a+(k+u)^{2} \natural a\right) f=\mathrm{Op}\left(H_{0} \natural a\right) f .
$$




\section{References}

[1] W. Arendt, C.J.K. Batty, M. Hieber, F. Neubrander, Vector-valued Laplace transforms and Cauchy problems, Birkhäuser (2001).

[2] N. Berglund, H. Kunz, Integrability and ergodicity of classical billiards in a magnetic field, J. Stat. Phys. 83, 81-126 (1996).

[3] C. Buchendorfer, PhD thesis, in preparation.

[4] M. Büttiker, Absence of backscattering in the quantum Hall effect in multiprobe conductors, Phys. Rev. B 38, 9375-9389 (1988).

[5] S. De Bièvre, J.V. Pulé, Propagating edge states for a magnetic Hamiltonian, Math. Phys. Electr. J. 5 (1999).

[6] P. Exner, P. Seba, Bound states in curved quantum waveguides, J. Math. Phys. 30, 2574-2579 (1989).

[7] J. Fröhlich, G.M. Graf, J. Walcher, On the extended nature of edge states of quantum Hall Hamiltonians, Ann. H. Poincaré 1, 405-442 (2000).

[8] K. Hornberger, U. Smilansky, Magnetic edge states, Phys. Rep. 367, 249-385 (2002).

[9] W. Hunziker, I.M. Sigal, The quantum N-body problem, J. Math. Phys. 41, 3448-3510 (2000).

[10] R.G. Littlejohn, W.G. Flynn, Geometric phases in the asymptotic theory of coupled wave equations, Phys. Rev. A 44, 5239-5256 (1991).

[11] A. Martinez, An introduction to semiclassical and microlocal analysis, Springer (2002).

[12] G. Panati, H. Spohn, S. Teufel, Space-adiabatic perturbation theory, Adv. Theor. Math. Phys. 7, 145-204 (2003).

[13] M. Reed, B. Simon, Methods of modern mathematical physics, vol. 3, Academic Press (1979).

[14] D.R. Yafaev, Mathematical scattering theory, American Mathematical Society (1992).

Christoph Buchendorfer and Gian Michele Graf

Institut für Theoretische Physik

ETH-Hönggerberg HPZ

CH-8093 Zürich, Switzerland

email: cbuchend@itp.phys.ethz.ch

email: gmgraf@itp.phys.ethz.ch

Communicated by Yosi Avron

submitted 23/02/05, accepted 3/05/05 